\title{
Superposition Method for Force Estimations on Bodies in Supersonic and Hypersonic Flows
}

\author{
Ansgar Marwege,,$*$ Sebastian Willems,, \pm and Ali Gülhan \\ DLR, German Aerospace Center, 51147 Cologne, Germany \\ and \\ Michael J. Aftosmis and Eric C. Stern! \\ NASA Ames Research Center, Moffett Field, California 94035
}

\section{DOI: $10.2514 / 1 . A 34128$}

\begin{abstract}
Aerodynamic interactions of fragments of meteoroids and space debris during atmospheric entry influence the flight trajectories of the fragments and their strewn field on the Earth's surface as well as the meteoroids' airburst altitude and energy deposition rate in the atmosphere and thus the resulting ground damage. For the design of experiments in the Hypersonic Wind Tunnel H2K of DLR, German Aerospace Center in Cologne to investigate the interaction phenomena, a method is developed to estimate the forces on two interacting bodies in supersonic and hypersonic flows, which is more accurate than existing analytical and semi-analytical methods and more efficient than numerical simulations regarding the computational effort. It is based on the superposition of flowfields taken from a database of numerical simulations of a single body for a range of Mach numbers. Furthermore, three-dimensional computations of configurations of interacting bodies are carried out by NASA Ames Research Center. Results obtained with the superposition method are compared to the simulations by NASA and to results of computations that can be found in the literature. The results from the method show good agreement with the computations.
\end{abstract}

\section{Nomenclature}

$a_{x_{1}} \quad=$ streamwise acceleration of first sphere, $\mathrm{m} / \mathrm{s}^{2}$

$C_{D} \quad=$ aerodynamic coefficient for drag

$C_{L} \quad=$ aerodynamic coefficient for lift

$C_{2}=$ center of second sphere

$D=$ drag

$d \quad=$ diameter, $\mathrm{m}$

$F \quad=$ force

$F_{p}=$ pressure force

$F_{\tau}=$ force resulting from shear stress

$g=$ gravitational acceleration, $\mathrm{m} / \mathrm{s}^{2}$

$L \quad=$ lift

$M \quad=$ Mach number

$P \quad=\quad$ point on surface of second sphere

$p \quad=$ pressure

$R=$ specific gas constant for air

$R e \quad=$ Reynolds number

$R_{s} \quad=$ radius of bow shock

$r \quad=$ radius, $\mathrm{m}$

$T=$ temperature, $\mathrm{K}$

$v \quad=$ velocity, $\mathrm{m} / \mathrm{s}$

$x, y, z=$ Cartesian coordinates

$\beta=$ shock angle, deg

$\gamma=$ heat capacity ratio

Received 25 October 2017; revision received 14 February 2018; accepted for publication 17 April 2018; published online Open Access 7 August 2018. Copyright $\odot 2018$ by DLR e.V. (German Aerospace Center). Published by the American Institute of Aeronautics and Astronautics, Inc., with permission. All requests for copying and permission to reprint should be submitted to CCC at www.copyright.com; employ the ISSN 0022-4650 (print) or 1533-6794 (online) to initiate your request. See also AIAA Rights and Permissions www.aiaa.org/randp.

*Research Scientist, Supersonic and Hypersonic Technology Department, Institute of Aerodynamics and Flow Technology; ansgar.marwege @ dlr.de.

${ }^{\dagger}$ Research Scientist, Supersonic and Hypersonic Technology Department, Institute of Aerodynamics and Flow Technology; sebastian.willems@dlr.de.

${ }^{\ddagger}$ Head of Department, Supersonic and Hypersonic Technology Department, Institute of Aerodynamics and Flow Technology; ali.guelhan@dlr.de.

${ }^{\S}$ Aerospace Engineer, Computational Aerosciences Branch; michael. aftosmis@nasa.gov. Associate Fellow AIAA.

"Research Engineer, Thermal Protection Materials Branch; eric.c.stern@ nasa.gov. Member AIAA.

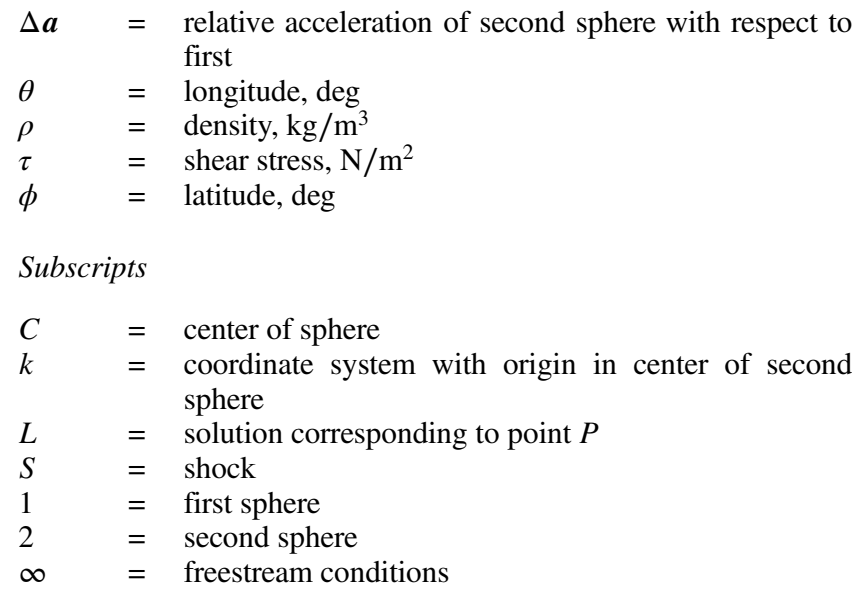

\section{Introduction}

D URING atmospheric entry, meteoroids may be disrupted into multiple fragments. The fragments that survive the ablation effect of the atmosphere interact aerodynamically in supersonic and hypersonic flows. Smaller fragments can follow larger ones without further fragmentation for a long distance [1,2]. Aerodynamic interactions also occur during the reentry of man-made space debris. The interaction phenomena can greatly influence the flight trajectories of the fragments of meteoroids and space debris and therefore their strewn field on the Earth's surface [3] . Furthermore, the rate of energy deposition into the atmosphere by a meteoroid and its airburst altitude can be highly sensitive to the interaction phenomena [4]. The energy deposition in the form of thermal radiation and blast waves can cause significant ground damage [4,5]. Therefore, understanding these interaction phenomena is indispensable for assessing potential ground damage from meteoroids and space debris.

Wind tunnel experiments for the investigation of these interaction phenomena are performed in the Hypersonic Wind Tunnel H2K at the Supersonic and Hypersonic Technologies Department of the Institute of Aerodynamics and Flow Technologies of DLR, German Aerospace Center. Furthermore, numerical computations are performed at NASA Ames Research Center. The high-quality experimental and numerical data are expected to improve the accuracy of the prediction of strewn fields, energy deposition rates 
and airburst altitudes, and hence the ground damage assessment of meteoroids and space debris.

An efficient method for approximating the forces on two interacting bodies is required to predict their trajectories for wind tunnel experiments. Numerically calculated flowfields for configurations of two bodies in supersonic or hypersonic flow can take significant computational effort. Flight trajectories require a broad range of configurations of the two bodies due to the changing relative position of the bodies along the trajectory and due to the changing freestream conditions, which further increases the computational effort required. For spherical bodies, there are an analytical method [1, $\underline{6}-\underline{8}]$ and a semianalytical method [9], which can efficiently approximate a broad range of configurations without the need of extensive computational effort. However, in these models, simplifications and assumptions that limit their range of validity and their accuracy are made.

In this Paper, a method is developed that is based on the superposition of numerically calculated flowfields of a single body to approximate the interaction phenomena of two bodies in supersonic and hypersonic flows without the need of the high computational effort of the simulation of all configurations of the two bodies. The method aims to efficiently approximate a broad range of configurations. As the method is not based on the same simplifications and assumptions as the analytical and semi-analytical methods, it is expected to be more accurate. The experiments in the Hypersonic Wind Tunnel H2K themselves are not described in this Paper.

For the current Paper, the two interacting bodies are approximated as spheres. This choice is advantageous as it ensures the comparability of results obtained with the method with previous research on spheres by other investigators. Additionally, it simplifies the interaction phenomena as the rotation of the interacting bodies can be neglected. Furthermore, in meteoroid entry trajectory simulation codes, it is typical to approximate the meteoroid fragments as spheres [3-ㅗㄱ. The method is developed mainly for the approximation of flight trajectories in the wind tunnel experiments. Nevertheless, it could also be applied for the prediction of flight trajectories of bodies entering the Earth's atmosphere.

NASA's Cart3D software is a Cartesian mesh-based inviscid computational fluid dynamics package aimed at flows over complex geometry that uses adjoint-based mesh adaptation to automatically refine the mesh to control the discretization error [10-12]. The solver is capable of computing multiple configurations of interacting bodies. While the computational effort is larger than for the superposition method, results can be expected to be more accurate since the analysis is performed over the full multibody configuration. A comparison of results obtained with the superposition method with the numerical computations can show which simplifications can be made to model interactions between multiple bodies in supersonic and hypersonic flows. Or, put in another way, critical configurations can be identified which need to be examined with more sophisticated viscous unsteady simulations and experiments.

The Paper is laid out as follows. First, the phenomena occurring for two spheres in supersonic and hypersonic flows are summarized. Then, after a short summary of existing approximation methods for the forces on the spheres, the superposition method is derived. The results obtained with the superposition method are compared to the results obtained by the numerical simulations and to results found in the literature. In addition, the superposition method is compared to the existing approximation methods.

\section{Two Spheres in Supersonic and Hypersonic Flows}

To approximate the interaction phenomena of multiple bodies in supersonic and hypersonic flows, the problem is reduced to the interaction of two spheres. Depending on the position of the second sphere relative to the first one, the inflow conditions for the second sphere vary.

A first sphere is positioned in supersonic or hypersonic flow. A second sphere is positioned downstream of the first sphere. The spheres have no spin. The geometric definitions are illustrated in Fig. 1. The downstream displacement of the second sphere is $x_{C_{2}}$, measured from the center of the first sphere to the center of the second

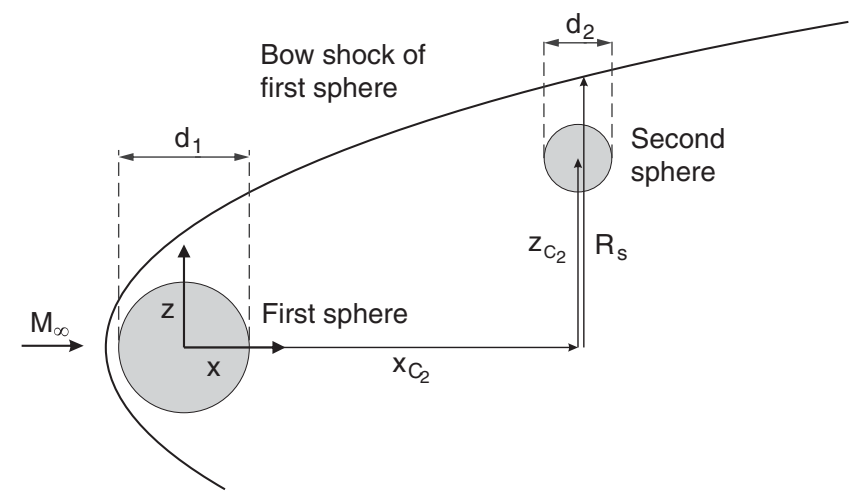

Fig. 1 Definition of the configuration of the two spheres (similar to Ref. [7]).

sphere. The $x$ axis is oriented in the freestream direction. The lateral displacement $z_{C_{2}}$ is the distance from the center of the first sphere to the center of the second one, normal to the inflow direction. $R_{s}$ is the radius of the undisturbed bow shock of the first sphere at $x_{C_{2}}$. The radii of the spheres are $r_{1}$ and $r_{2}$, and the diameters are $d_{1}$ and $d_{2}$.

The phenomena that can occur for a two-sphere configuration are shown in Fig. 2 and will be explained in the following. $\Delta \boldsymbol{a}$ is the total acceleration of the second sphere relative to the first one. It can be divided into its axial and lateral components $\Delta a_{x}$ and $\Delta a_{z}$.

If the second sphere is close to the region enclosed by the bow shock of the first sphere, it can either be expelled from or entrained in this region. If the relative lateral acceleration is large with respect to the relative axial acceleration, the sphere is expelled (see Fig. 2a); if it is small with respect to the relative axial acceleration or negative, the sphere is entrained (see Fig. 2b) (analogous to [7]).

The expansion and separation of the flow around the first sphere significantly reduces the static and dynamic pressure in the wake of the first sphere. This leads to a reduction in the drag of the second sphere and therefore to a negative $\Delta a_{x}$. So the first sphere drafts the second one, and the second sphere moves toward the first (see Fig. 2c).

If the second sphere is positioned in such a way that the first bow shock impinges on the surface of the sphere, a phenomenon called shock surfing or shock wave surfing can occur $[7,14]$. If the relative acceleration of the second sphere is tangential to the bow shock of the first sphere, it follows the shape of the bow shock and "surfs" on it. Laurence and Deiterding [1] showed that for a static first body shock wave surfing occurs if the force on the second body is tangential to the shock of the first body. This results in large lateral velocities [1]. More detailed dynamic analysis of this phenomenon can be found in Refs. [1,8].

Which of these phenomena occurs depends strongly on the configuration of the two spheres and on the freestream conditions, particularly the Mach number. The parameters of the configurations are the radius ratio of the spheres and their densities, the downstream and lateral displacement of the second sphere relative to the first, and the initial relative velocities of the second sphere with respect to the first sphere.

\section{Previous Methods for Calculation of Multibody Interactions}

Approaches for the calculation of multibody interactions are summarized in the following. Investigations on the interactions of multiple bodies in hypersonic flows were carried out through the investigation of terrestrial strewn fields for meteoroid fragments by Passey and Melosh [3]. Based on this research, the interaction phenomenon was modeled numerically by Artem'eva and Shuvalov $[2,14]$. These numerical models, however, are lacking accuracy as discovered by Boroviĉka and Kalenda [15]. In Refs. [1, $\underline{6}-\underline{8}]$, this research was followed by a combination of numerical, experimental, and analytical investigations. Prévereaud [9] further developed the analytical model of Laurence [6] to a semi-analytical model. 


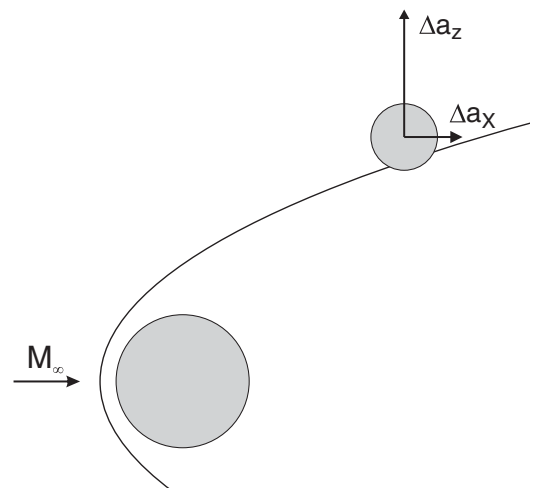

a)

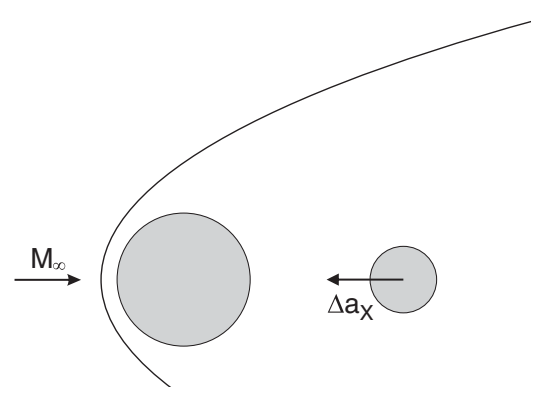

c)

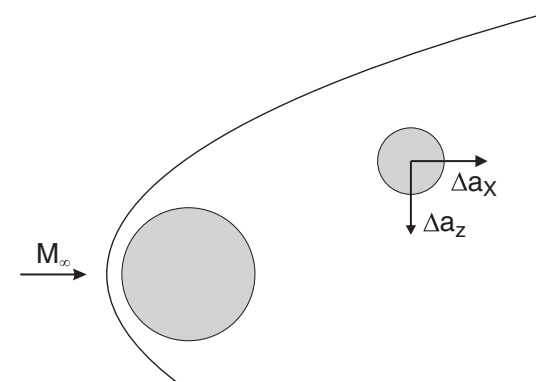

b)

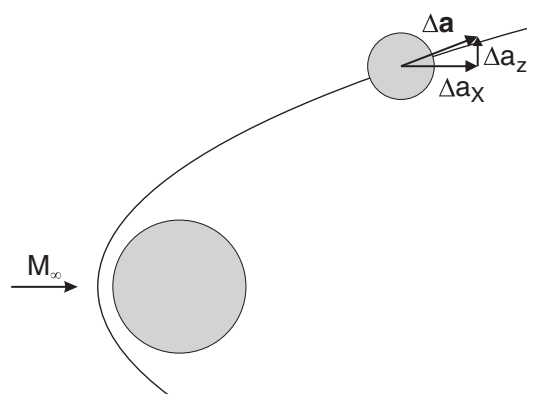

d)

Fig. 2 Phenomena occurring for two-sphere interactions (similar to Ref. [19]): a) expulsion, b) entrainment, c) drafting, and d) shock wave surfing.

In Ref. [6], an analytical model was developed for the description of the forces on the second sphere. In Ref. [7], this model was partly summarized and slightly changed. The method presented in Ref. [7] is independent of the freestream Mach number, and it is only valid if the second sphere is positioned completely inside the region enclosed by the bow shock of the first sphere. In Ref. [6], also, a method to take into account the shock of the first sphere impinging on the second sphere was developed. It was further validated in Ref. [1]. This method depends on the freestream Mach number. The analytical method is summarized shortly in the following, and its principle is shown in Fig. 3a.

The pressure distribution downstream of the first sphere in the shocked region is determined with the blast wave analogy. It is used to determine the inflow conditions for the second sphere (see Fig. $\underline{3 a}$ ). The axial and lateral variation of the stagnation pressure over the surface of the second sphere is taken into account by a Taylor series [6,7]. The pressure distribution on the second sphere is modeled as a modified Newtonian pressure distribution.

Furthermore, the shock of the first sphere is impinging on the second one. The shock-shock interactions and their influence on the pressure distribution on the surface of the second sphere are assumed to be negligible. In Ref. [1], this is argued to be reasonable as the spatially very limited influence of the pressure peak arising from the impingement only contributes a little to the integral of the pressure over the surface of the sphere for the calculation of the forces. The shock of the first sphere is assumed to solely serve to devide the flow around the second sphere in two regions, the region enclosed by the bow shock of the first sphere and the freestream region.

It is expected that inaccuracies will arise from the simplifications introduced by the blast wave analogy as well as from neglecting shock-shock interactions. Laurence [] stated that, especially in the wake region of the first sphere, the model does not apply as the blast wave analogy does not cover this region. Also, the separation shock emerging from the separation of the boundary layer from the first sphere is absent in the blast wave analogy and is thus not considered in the model. Prévereaud [9] replaced the flowfield in the shocked region obtained with the blast wave analogy by a numerically calculated reference field. This is shown in principle in Fig. 3b. Inaccuracies arise for both methods from the use of the modified Newtonian pressure distribution especially in regions of small Mach numbers as it is only valid for high Mach numbers [16].

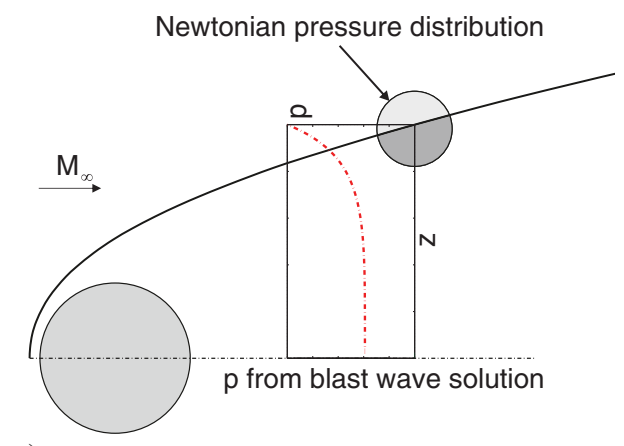

a)

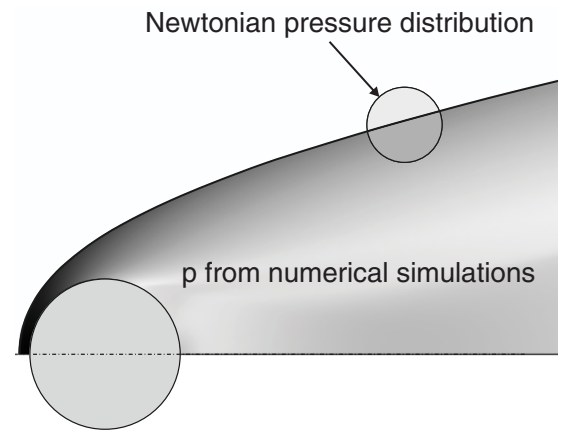

b)

Fig. 3 a) Analytical method developed in Refs. [1, $\underline{6}-\underline{8}]$ and b) semi-analytical method developed in Ref. [9] for the calculation of the interaction of two spheres in hypersonic flows. 
In the current Paper, a method that is a consequent continuation of the analytical and semi-analytical method is presented. In this method, the flowfield around the first sphere and the pressure distribution on the surface of the second sphere are both obtained from numerical simulations. Therefore, the inaccuracies from the blast wave solution and the Newtonian pressure distribution can be reduced. The principle will be discussed in more detail in Sec. $\underline{\text { V. }}$

\section{Numerical Tools}

\section{A. DLR Flow Solver TAU}

All simulations of flowfields used with the superposition method are performed with the flow solver TAU $[17,18]$, developed by DLR Institute of Aerodynamics and Flow Technology in Braunschweig and Götingen, Germany. It can solve numerically viscous and inviscid flows around complex geometries on structured, unstructured, and hybrid grids for subsonic up to hypersonic flow regimes and features adaptive mesh refinement to resolve important flow features.

\section{B. NASA Flow Solver Cart3D}

NASA's Cart3D simulation package uses a Cartesian cut-cell approach in which the Euler equations are discretized on a multilevel Cartesian mesh with embedded boundaries $[10,11]$. The meshes consist of regular Cartesian hexahedra everywhere, except for a layer of body-intersecting cut cells at the boundaries. Although the mesh consists of nested Cartesian cells, it is viewed as an unstructured collection of control volumes, making the approach well suited for parallel computation and solution-adaptive mesh refinement. Steadystate flow solutions are obtained using a five-stage Runge-Kutta scheme with local time stepping and a multigrid. Domain decomposition via space-filling curves permits parallel computation with excellent scalability. Meshes are automatically generated using adjoint-based adaptation. The duality-preserving discrete adjoint leverages the domain decomposition and other infrastructure from the primal solver and provides both error estimation and output-driven mesh adaptation [12]. For the cases discussed here, each simulation used the package's adaptive meshing capability, and the adaptation goal was to reduce the discretization error in computing loads on the spheres.

\section{Superposition Method}

In this section, the superposition method is developed. The input for the method is a database, the freestream Mach number, and the relative position of the second to the first sphere. Using the database, the superposition method approximates the aerodynamic forces on the second sphere. The effect of the presence of a second sphere on the first sphere is neglected by the method. This is reasonable, as for high supersonic flows the region downstream of the first sphere is mainly supersonic and no information can propagate upstream in supersonic flows. If the second sphere is located in the subsonic wake region of the first sphere, this assumption fails.

The principle of the method is as follows. First, the database is computed. This database contains simulations of the flowfield of one sphere for a range of Mach numbers. The Reynolds number is kept constant. Then, two-sphere configurations are calculated. The flowfield for the first sphere is taken from the database. A second sphere is positioned in the flowfield of the first sphere. The Mach number, pressure, and flow direction in the flow solution of the first sphere are interpolated onto the surface of the second sphere. Figure $4 \mathrm{a}$ shows this for the Mach number. Then, each point on the sphere is treated independently. In the following, an arbitrary point $P_{1}$ on the surface of the sphere is considered. The flowfield for the first sphere and for an arbitrary point $P_{1}$ on the second sphere is sketched in Fig. 4c. The inflow Mach number of the point $M_{\infty, P_{1}}$ and its inflow pressure $p_{\infty, P_{1}}$ are taken from the flow solution of the first sphere. The inflow conditions for this point are considered to be valid for the complete second sphere. Also, the local inflow angle is considered. The position of the point is not determined relative to the freestream but relative to the local inflow. With the position of the point relative to the local inflow and with the local inflow conditions $M_{\infty, P_{1}}$ and $p_{\infty, P_{1}}$, the pressure and the shear stress at the surface of the sphere for this point are taken from the database. Thus, the pressure and shear stress at $P_{1}$ are calculated as if the sphere was positioned in a freestream with the local inflow angle and with $M_{\infty, P_{1}}$ and $p_{\infty, P_{1}}$. This procedure is carried out for all points on the surface of the sphere. The flowfield for the complete sphere is a superposition of the independent flowfields for the points on the sphere. The superposition of the flowfields is shown schematically for three

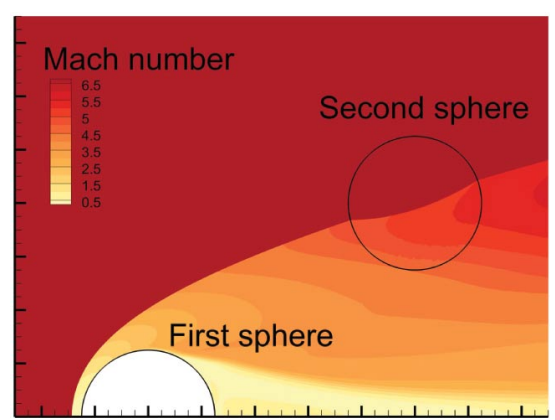

a)

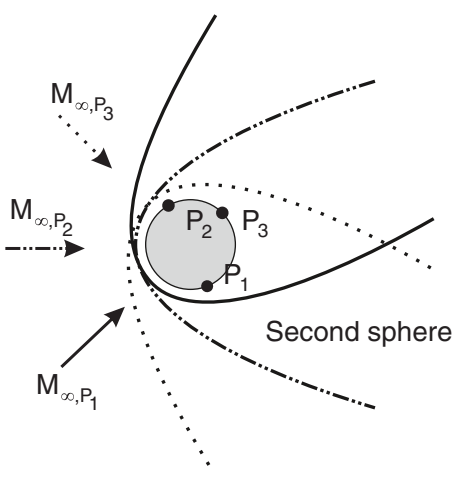

b)

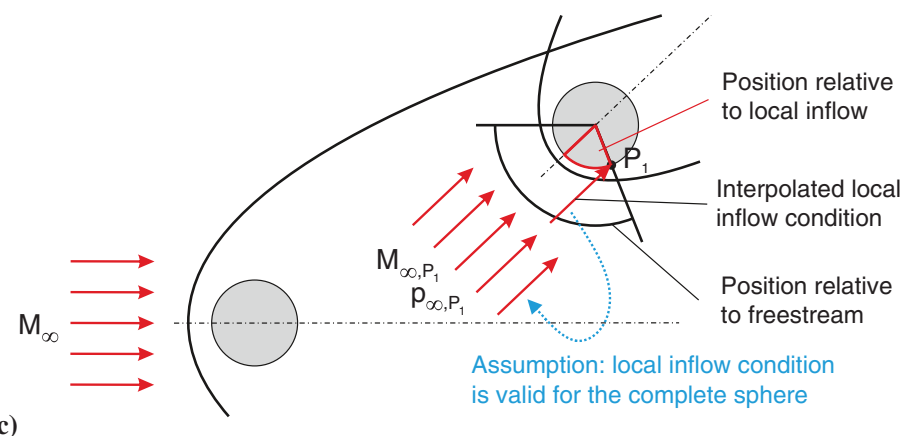

Fig. 4 Principle of the superposition method: a) inflow conditions for the points on the second sphere from the flow solution of the first sphere, b) independent flowfields for several points on the second sphere, and c) flowfield of the first sphere and of the point $P_{1}$ on the second sphere. 
Table 1 Reference parameters for the database

\begin{tabular}{lc}
\hline \hline Reference parameter & Value \\
\hline Freestream pressure $p_{\infty}, \mathrm{Pa}$ & 136.44 \\
Freestream temperature $T_{\infty}, \mathrm{K}$ & 46.52 \\
Freestream velocity $v_{\infty}, \mathrm{m} / \mathrm{s}$ & 958.7 \\
Freestream density $\rho_{\infty}, \mathrm{kg} / \mathrm{m}^{3}$ & 0.01022 \\
Mach number $M_{\infty}$ & 7.012 \\
Heat capacity ratio $\gamma$ & 1.4 \\
Specific gas constant for air $R, \mathrm{~J} /(\mathrm{kg} \cdot \mathrm{K})$ & 287 \\
Unit Reynolds number $R e_{u}, \mathrm{~m}^{-1}$ & $3.32 \cdot 10^{6}$ \\
Sphere diameter $d, \mathrm{~m}$ & 0.05 \\
Temperature on the surface of the sphere $T, \mathrm{~K}$ & 293.15 \\
\hline \hline
\end{tabular}

points $P_{1}, P_{2}$, and $P_{3}$ in Fig. $4 \mathrm{~b}$. For the demonstration of the principle, the inflow angles shown are chosen to be clearly different in this figure. The aerodynamic forces on the sphere are calculated by integrating the pressure and the shear stress obtained for the points over the complete surface of the sphere.

\section{A. Database of Numerical Simulations}

In this subsection, the database used for the calculations with the superposition method is described. In the Introduction, it was stated that the superposition method shall provide data for the estimation of interaction phenomena in wind tunnel experiments. The experiments at the Supersonic and Hypersonic Technologies Department of DLR in Cologne are performed in the $\mathrm{H} 2 \mathrm{~K}$ wind tunnel [19]. For the database, reference parameters that are easy to realize in the wind tunnel experiments are chosen. These reference parameters are shown in Table 1.

The superposition method is developed for configurations of two bodies in supersonic and hypersonic flows. The highest Mach number that can be reached in the wind tunnel is $M_{\infty}=11.2$. Therefore, the highest Mach number of the database is $M_{\infty}=12$. Although the freestream is always super- or hypersonic, the inflow for the second sphere can be sub- or transonic, e.g., in the wake of the first sphere. Therefore, the database covers the range $0.1 \leq M_{\infty} \leq$ 12.0 with a step size of $\Delta M_{\infty}=0.1$.

\section{Physical Conditions and Assumptions}

For simplicity of the model, the flow is assumed to be completely laminar and steady. Particularly in the subsonic wake region of the first sphere, this assumption does not hold. However, the region that is affected by turbulence is small, particularly for high Mach numbers. A small turbulent wake region also justifies assuming an axisymmetric flow. Therefore, the simulations are carried out as two-dimensional (2D) axisymmetric.

For viscous flows, the similarity parameters Mach number $M$ and Reynolds number $R e$ have to be considered. Measurements by Naumann [20], described in Ref. [21], showed that for Mach numbers higher than 0.8 the drag coefficient of spheres is independent from the Reynolds number. Thus, the Reynolds number is set constant for the database.

In this Paper, high-temperature effects resulting from hypersonic flows are neglected. As explained in the Introduction, the method is developed to provide approximate calculations of two-sphere configurations for flight trajectories during wind tunnel experiments. The experiments are performed in a cold gas wind tunnel, where high-temperature effects will not occur even for high Mach numbers. Consequently, the high-temperature effects are also neglected in the simulations used in the superposition method, and an ideal gas is assumed. The database can be easily exchanged, if these effects should be considered for other applications.

\section{Numerical Setup}

For the numerical simulations, the second-order AUSMDV upwind scheme [22] was chosen for the spatial discretization. To account for the differences in the requirements for supersonic and subsonic flows, two different computational domains were used, one for super- and hypersonic flows and another one for sub- and transonic flows. They are shown in Figs. $5 \mathrm{a}$ and $5 \mathrm{~b}$, respectively. The shape of the inflow boundary of the supersonic domain is hyperbolic. This ensures that the bow shock remains inside the inflow boundary and exits from the outflow boundary. Furthermore, this shape minimizes the grid points to be calculated. The grid was adapted to the flow with the TAU adaptation tool. For the sub- and transonic computations, a circular domain with a radius of 100 sphere diameters was chosen. Five passes of adaptation for the super- and hypersonic grid resulted in about 211,000 cells, and six passes for the sub- and transonic grid resulted in about 192,000 cells.

The assumptions for the calculations were a laminar, steady, and axisymmetric flow. For the unit Reynolds number of $R e_{u_{\infty}}=3.32 \cdot 10^{6} \mathrm{~m}^{-1}$ and the diameter of the sphere of the reference configuration $d=0.05 \mathrm{~m}$ (see Table 1 ), the model Reynolds number was $R e_{\infty}=1.66 \cdot 10^{5}$. Therefore, in the subsonic regime, the assumption of laminar flow was valid, since the Reynolds
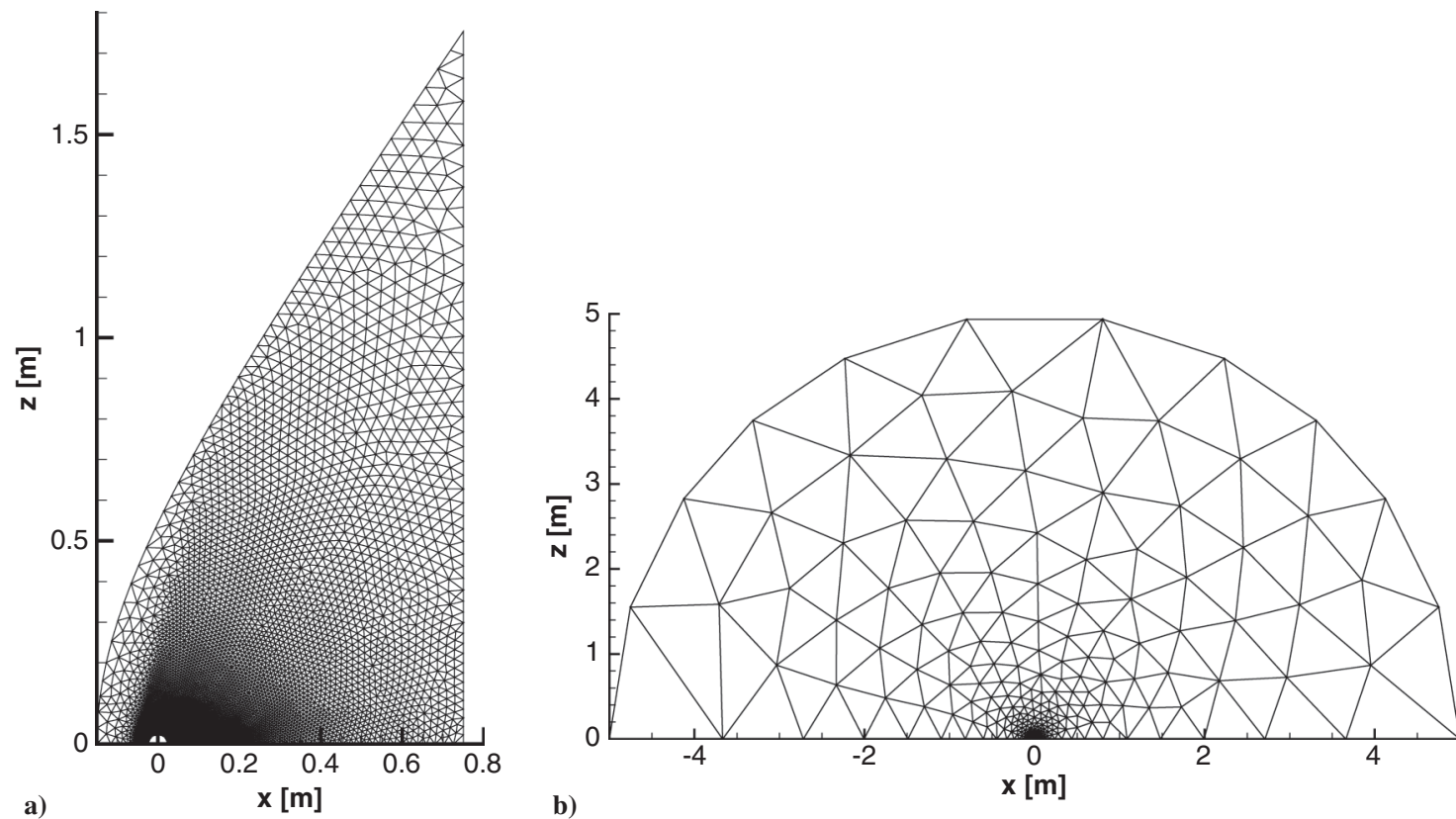

Fig. 5 Computational domain a) for the super- and hypersonic regimes and b) for the sub- and transonic regimes. 
number for the transition of laminar to turbulent flow is approximately $R e_{\infty}=2 \cdot 10^{5}$ for spheres in subsonic flows [21]. The assumptions of steady and axisymmetric flow, however, are not valid in subsonic regimes for Reynolds numbers $R e_{\infty}>200$ [21]. Therefore, the flow solutions in the subsonic regime were not very accurate. However, almost the complete flowfield around the first sphere was supersonic, and therefore usually the complete inflow of the second sphere was supersonic or just small parts were subsonic. Hence, the error in the calculation of the forces on the second sphere due to inaccuracies in the subsonic simulations was small.

As mentioned before, the database can be easily exchanged. So, the $2 \mathrm{D}$ calculations can be replaced by more sophisticated calculations or by data from experiments at a later point in time.

\section{B. Geometric Definitions}

In this subsection, the geometric definitions for the two-sphere configurations are defined. An initial Cartesian coordinate system is used to define the relative position of the two spheres (see Fig. 6a). The origin of the coordinate system is located in the center of the first sphere. The $x$ axis is aligned with the flow direction. The $z$ axis is oriented in the opposite direction of the gravitational acceleration $g$. The $y$ axis is oriented normal to the $x-z$ plane so that the axes form a right-handed coordinate system. Figure 6 a shows a sketch of the second sphere with the center $C_{2}\left(x_{C_{2}}, y_{C_{2}}, z_{C_{2}}\right)$.

The points on the second sphere are defined in a Cartesian coordinate system with its origin located in the center of the second sphere. The index of this coordinate system is $k$. The $x_{k}$ axis is aligned with the flow direction and is parallel to the $x$ axis. The $z_{k}$ axis is orthogonal to the $x$ axis and points from it to $C_{2}$ (see Fig. 6a). The $x_{k}-y_{k}-z_{k}$ coordinate system is right handed. The angle between the $z$ and $z_{k}$ axes is $\psi$. The $k$-coordinate system with respect to the initial coordinate system is shown in Fig. 6 b.

Additionally, the points on the second sphere can be defined in spherical coordinates with $r_{2}, \theta$, and $\phi$, where $r_{2}$ is the radius of the second sphere and $\theta$ and $\phi$ are the longitude and the latitude regarding the $k$-coordinate system. This is sketched in Fig. $6 \mathrm{c}$. The longitude $\theta$ is positive in the mathematical positive sense around the $x_{k}$ axis, starting in the $y_{k}-z_{k}$ plane. The latitude $\phi$ is positive for positive $x_{k}$ values and negative for negative $x_{k}$ values. In Fig. $\underline{6 c}$, a point with a positive $\theta$ and a negative $\phi$ is sketched.

\section{Determination of State Functions on Surface of Second Sphere}

In this subsection, the procedure for obtaining the state functions on the surface of the second sphere is discussed. In Sec. V, it was explained that the inflow conditions for the points on the second sphere are taken from the flow solution of the first sphere. The flowfield of the first sphere is calculated as axially symmetric (see Sec. V.A). Hence, the solution is two dimensional. It is calculated in the $x-z$ plane. Every point $L$ of the flow solution of the first sphere is valid on a circle around the $x$ axis. This is sketched in Fig. 7 . Therefore, any point $P\left(x_{P}, y_{P}, z_{P}\right)$ on the sphere corresponds to a

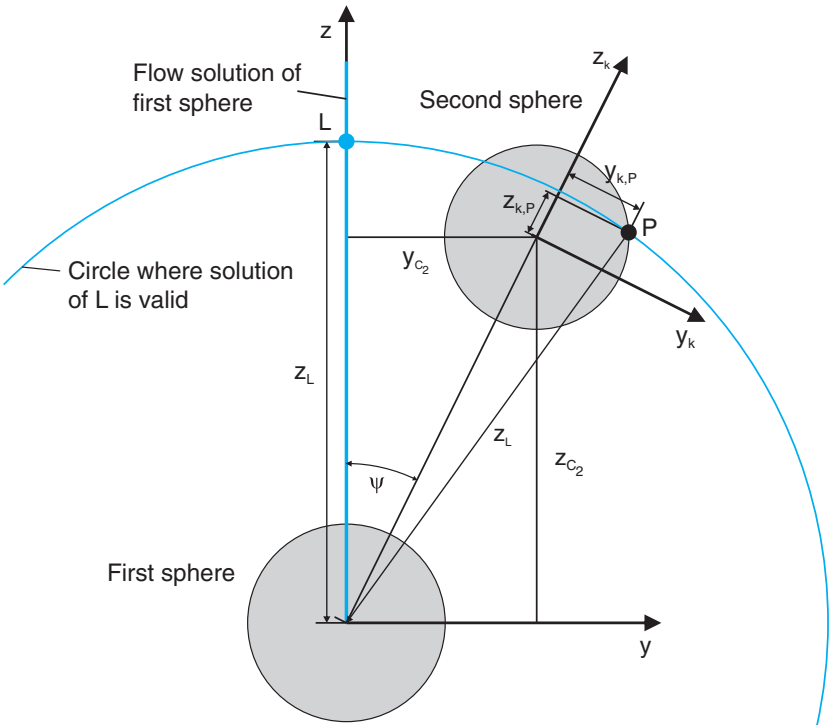

Fig. 7 Geometric dependencies of a point $P$ on the second sphere and the corresponding point $L$ in the flow solution of the first sphere.

point $L\left(x_{L}, 0, z_{L}\right)$ of the solution with $x_{L}=x_{P}$ and $z_{L}=\sqrt{y_{P}^{2}+z_{P}^{2}}$ (the geometric dependencies are sketched in Fig. 7).

The grid of the flow solution of the first sphere, grid A, and the grid of the points on the surface of the second sphere, grid $\mathrm{B}$, are superimposed. For every point $P$ of grid $\mathrm{B}$, the corresponding point $L$ in the solution plane is determined. Then, the flow variables of $L$ are interpolated onto $P$. Because of the axial symmetry of the flow solution of the first sphere, the points in the solution plane are determined for a half-sphere only, as the points with the same $x_{k, P}$ and $z_{k, P}$ correspond to the same $L$ for positive and negative $y_{k, P}$ values. The flow variables that are interpolated are the Mach number, the pressure, the density, and the velocity vector $\boldsymbol{v}_{L}=\left(v_{x, L}, v_{z, L}\right)^{T}$.

The accuracy of the inflow parameters for the points on the second sphere depends on the level of refinement of both grids.

The flow variables obtained from the interpolation are used together with the database of flow solutions to determine the pressure and the shear stress at the points on the surface of the second sphere. For that, the Mach number is used to determine the solutions from the database corresponding to the inflow conditions for the surface point of the sphere. The inflow angle is used to determine the point in the solutions corresponding to the grid point on the surface of the sphere. The pressure and skin friction are then interpolated from the solution points onto the surface point on the sphere. The pressure is scaled according to the local inflow pressure of the surface point.

\section{Forces Acting on Second Sphere}

Because of the symmetry of the flow solution of the first sphere with respect to the $x_{k}-z_{k}$ plane, the components of the forces on the

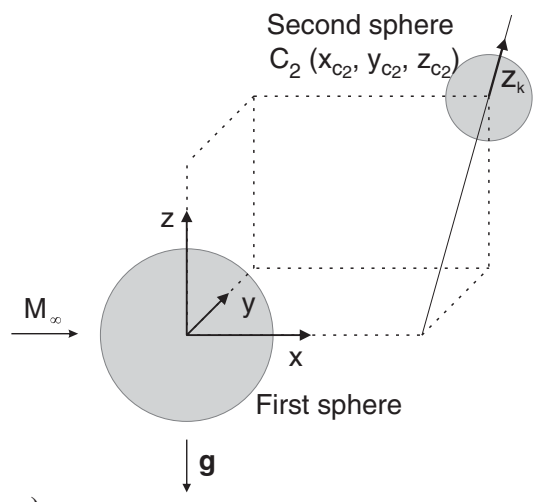

a)

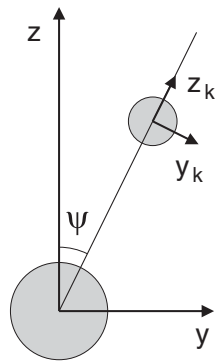

b)

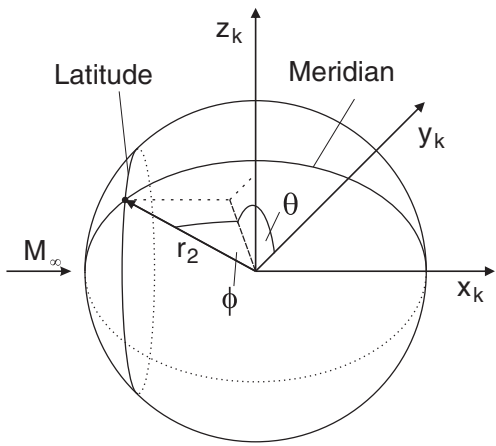

c)

Fig. 6 Geometric definitions: a) initial coordinate system, b) $k$-coordinate system with respect to the initial coordinate system, and c) spherical coordinates of surface points on the second sphere. 
second sphere in the $x_{k}$ and $z_{k}$ directions are the same for the halfsphere with positive $y_{k}$ or negative $y_{k}$. In the $y_{k}$ direction, the forces cancel out. Therefore, the forces are only calculated for a half-sphere.

Integrating the pressure and the shear stress over the surface of the sphere, the forces on the sphere are obtained. Summing up the pressure force $\boldsymbol{F}_{p}$ and the force resulting from the shear stress $\boldsymbol{F}_{\tau}$, the complete force on the sphere $\boldsymbol{F}$ is obtained,

$$
\boldsymbol{F}=\left(\begin{array}{l}
F_{x_{k}} \\
F_{y_{k}} \\
F_{z_{k}}
\end{array}\right)=\left(\begin{array}{c}
F_{p, x_{k}}+F_{\tau, x_{k}} \\
0 \\
F_{p, z_{k}}+F_{\tau, z_{k}}
\end{array}\right)
$$

The forces in the initial coordinate system are obtained using the angle of rotation $\psi$ (see Fig. 7):

$$
\boldsymbol{F}=\left(\begin{array}{c}
F_{x} \\
F_{y} \\
F_{z}
\end{array}\right)=\left(\begin{array}{c}
F_{x_{k}} \\
F_{z_{k}} \sin (\psi) \\
F_{z_{k}} \cos (\psi)
\end{array}\right)
$$

\section{Inviscid Computations of Multiple Bodies with Cart3D}

Figure $\underline{8}$ shows a sample of the inviscid simulation results performed with Cart3D. The figure shows contours of local density overlaid with the final adapted Cartesian mesh, which contained approximately 6 million cells. The mesh adaptation was driven using an objective function consisting of the loads on the two spheres, and the mesh adaptation sought to minimize error in this objective.

Ten passes of adaptive refinement resulted in a mesh with 6.2 million cells with very good mesh convergence of the integrated loads.

The figure shows an example at Mach 10 in which the aft sphere is half the diameter of the front $\left(d_{2} / d_{1}=1 / 2\right)$. The aft sphere is located four diameters behind the leading sphere, and the bow shock of the leading sphere is impinging upon it. To accurately compute the loads on the aft sphere, the mesh refinement focuses on the upper half of the leading sphere's shock system and uses very high resolution to resolve the shock-shock interactions in the bowshock of the aft sphere.

\section{Results}

\section{A. Verification of Superposition Method}

For verification of the superposition method, results obtained with the method are compared to results of three-dimensional (3D) computations with the Cart3D flow solver and with results of 3D computations by Laurence [6] with the AMROC software. Furthermore, results of one configuration with the superposition method are compared in detail to a laminar 3D computation with TAU.

\section{Comparison with $3 D$ Computations}

Laurence [6] carried out 3D computations for two-sphere configurations for several Mach numbers. In these computations, the three-dimensional Euler equations were solved for ideal gas. Laurence used the AMROC sofware [23], which is very similar to the Cart3D software.

In Figs. 9 and 10 , the values of lift coefficient $C_{L}$ and drag coefficient $\bar{C}_{D}$ obtained with the superposition method for different downstream and lateral displacements of the second sphere are compared to computations with the Cart3D computations described in Sec. VI and with the AMROC computations by Laurence [6] for $M_{\infty}=\overline{10}$. In addition, Figs. 9 and 10 contain the results of $3 \mathrm{D}$ computations by Laurence for $\bar{M}_{\infty}=\overline{50}$. Also, the solution obtained with the Mach number independent analytical method descibed in Ref. [7] is shown. In Ref. [6], a method to take into account the shock of the first sphere impinging on the second one was proposed. The solution for $M_{\infty}=50$ obtained by Laurence with this method is also presented in Figs. $\underline{9}$ and 10 . As $M_{\infty}=50$ is far out of the range of Mach numbers of the database, no solutions are calculated with the superposition method for this Mach number. Results obtained with the semi-analytical method shown for $M_{\infty}=10$ are taken from Ref. [9]. This data were approximated with smoothed curve fits. For the calculations with the superposition method, 90,000 points were used on the grid of half of the second sphere. The grid of the flowfield of the first sphere was adapted to the flow with the TAU adaptation tool. The position in the $z$ direction was normalized with the shock radius $R_{s}$ at the downstream position of the sphere $x_{C_{2}}$. For the data of the superposition method, the shock radius for the normalization was taken from the 2D flowfield of the first sphere. The data of the Cart3D computations and the 3D computations by Laurence were normalized with the shock radius obtained from the respective computations.

The $C_{D}$ and $C_{L}$ values of the Cart3D computations and the AMROC computations by Laurence [6] show very good agreement. This was expected, as the solvers have a similar architecture and both apply dynamic Cartesian mesh adaptation for inviscid conditions. Only in Fig. 9a, two points show slight deviations, the $C_{D}$ value at approximately $z_{C_{2}} / R_{s}=0.2$ and the $C_{L}$ value at approximately $z_{C_{2}} / R_{s}=1.07$.

In Figs. 9 and 10, two types of graphs are calculated with the superposition method. The red dashed graphs consider the forces resulting from the pressure and from the shear stress, and the continuous red graphs only consider the pressure forces. As the Cart3D computations and the computations by Laurence [6] only considered the pressure forces, the continuous curves of the superposition method lie closer to the computed solutions than the graphs that are also considering the shear stresses. Additionally, the graphs show that the contributions of the shear stress to the forces on the sphere are small. The agreement of the superposition method with the $3 \mathrm{D}$ computations is generally very good.

For the case in Fig. 9a with $x_{C_{2}} / d_{1}=1.5$ and $d_{2} / d_{1}=1 / 2$, the values for the maximum lift coefficient and the maximum drag coefficient resulting from the superposition method are larger than the values resulting from the $3 \mathrm{D}$ calculations. The maximum drag is larger by approximately $(1.6-1.4) / 1.4=14.3 \%$, and the lift is larger by $(0.5-0.4) / 0.4=25 \%$. The configuration of Fig. 9 a for a larger downstream displacement of the second sphere is shown in Fig. 9b;
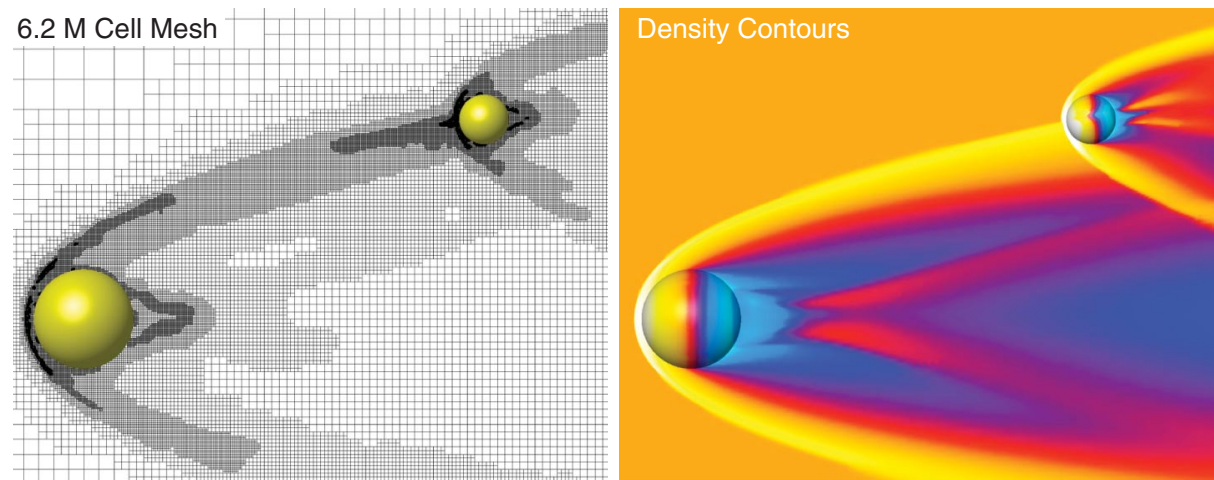

Fig. 8 Cart3D simulation showing adapted computational mesh and density contours in the resulting solution for Mach 10 flow over two spheres. The aft sphere is 4 diameters behind the front; $x_{C_{2}} / d_{1}=4, d_{2} / d_{1}=1 / 2$. 


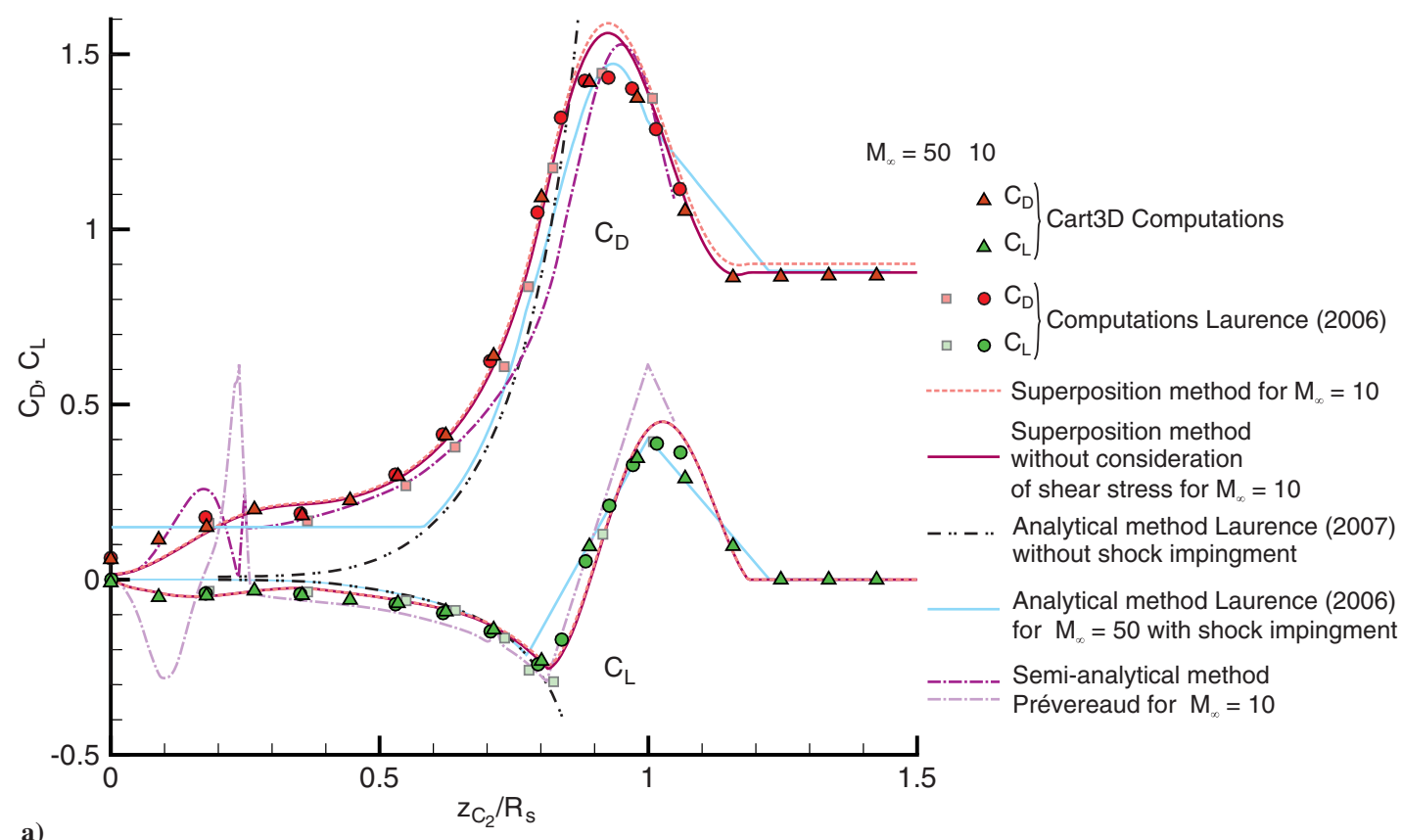

a)

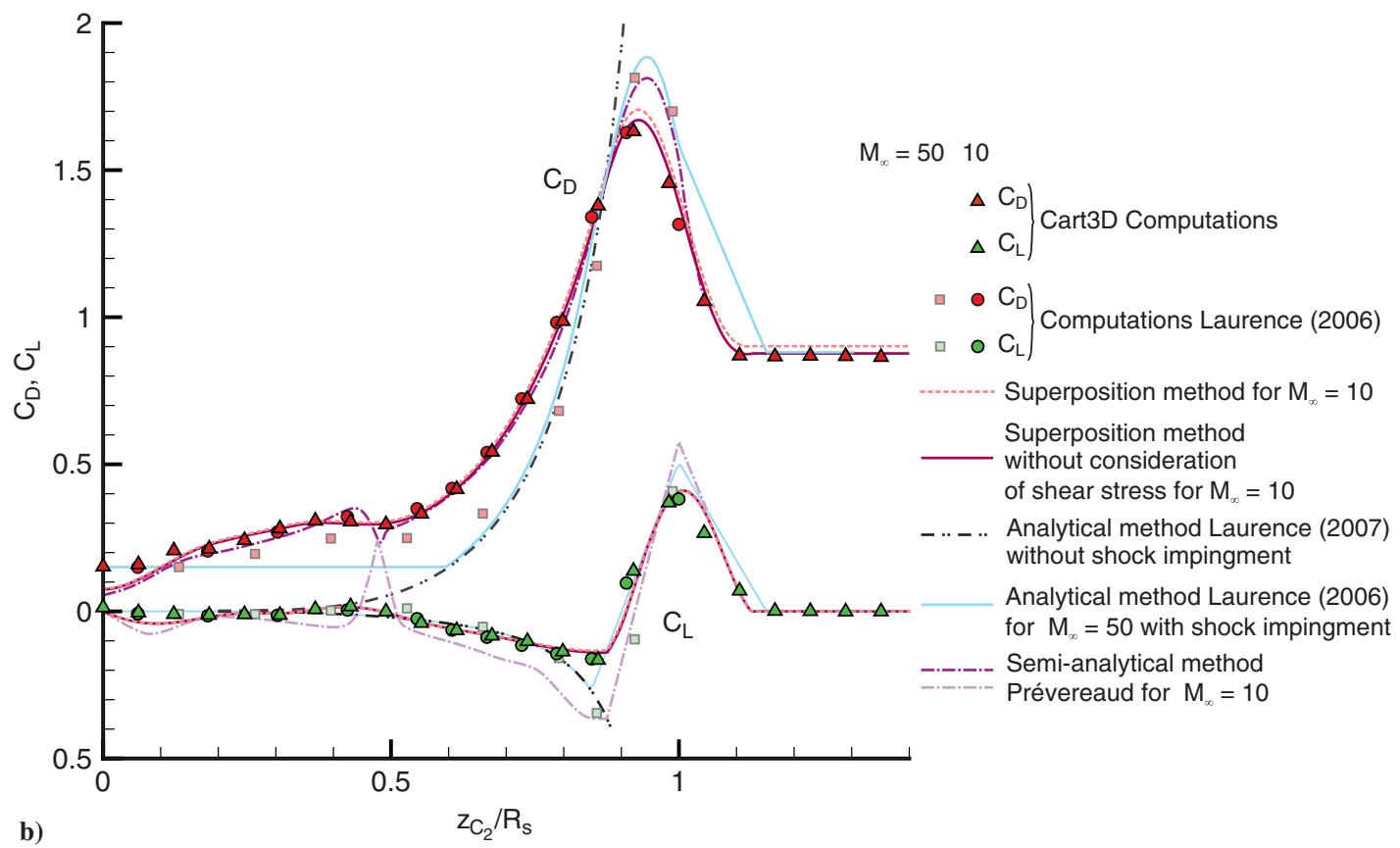

Fig. 9 Comparison of $C_{D}$ and $C_{L}$ values resulting from the superposition method, with computations with Cart3D for $M_{\infty}=10$, with AMROC computations for $M_{\infty}=10$ and $M_{\infty}=50$ by Laurence [6], with the Mach-number-independent analytical method of Ref. [7], with the analytical method considering the impingment for $M_{\infty}=50$ from Ref. [6] , and with the semi-analytical method for $M_{\infty}=10$ obtained from Ref. [9] : a) $x_{C_{2}} / d_{1}=1.5$, $d_{2} / d_{1}=1 / 2$; b) $x_{C_{2}} / d_{1}=4, d_{2} / d_{1}=1 / 2$.

the calculated graph matches the points very well, including the maximum values. The result of the same downstream displacement as in Fig. 9a but for a smaller diameter ratio can be seen in Fig. 10. As before, the curves match the points very well. From this analysis, it can be suggested that a larger downstream displacement and a smaller radius ratio favor the accuracy of the method.

In the region where the second sphere is positioned completely outside of the shock of the first sphere $\left(z_{C_{2}} / R_{s} \gtrsim 1.35\right.$ in Fig. 9a and $z_{C_{2}} / R_{s} \gtrsim 1.24$ in Fig. 9b), the $C_{D}$ values of the superposition method have to equal the $C_{D}$ of a sphere in the freestream at $M_{\infty}=10.0$. The difference of the $C_{D}$ of the superpositon method and the $C_{D}$ of the axisymmetric computation of the database of the superpositon method for $M_{\infty}=10.0$ is -0.0035 , which results in a relative deviation of $-0.39 \%$. In this region, the $C_{D}$ values of the superposition method are larger than the values computed with Cart3D. This is due to the fact that the database used for the superposition method in this Paper is calculated assuming laminar flow, while the Cart3D computations assume inviscid flow.

The solution of the Mach-number-independent analytical method of Laurence et al. [7] shows better agreement with the computations for $M_{\infty}=50$ than for $M_{\infty}=10$, as it assumes an infinite freestream Mach number. Therefore, as could be expected, a comparison of the solution obtained with the superposition method with the Machnumber-independent analytical method shows that the curves of the superposition method match the 3D computations better for $M_{\infty}=10$. Furthermore, as described in Sec. III, the Mach-number-independent analytical method is only valid if the second sphere is positioned completely in the region enclosed by the bow shock of the first sphere.

Hence, also, results obtained with the analytical method that models the bow shock of the first sphere impinging on the second 


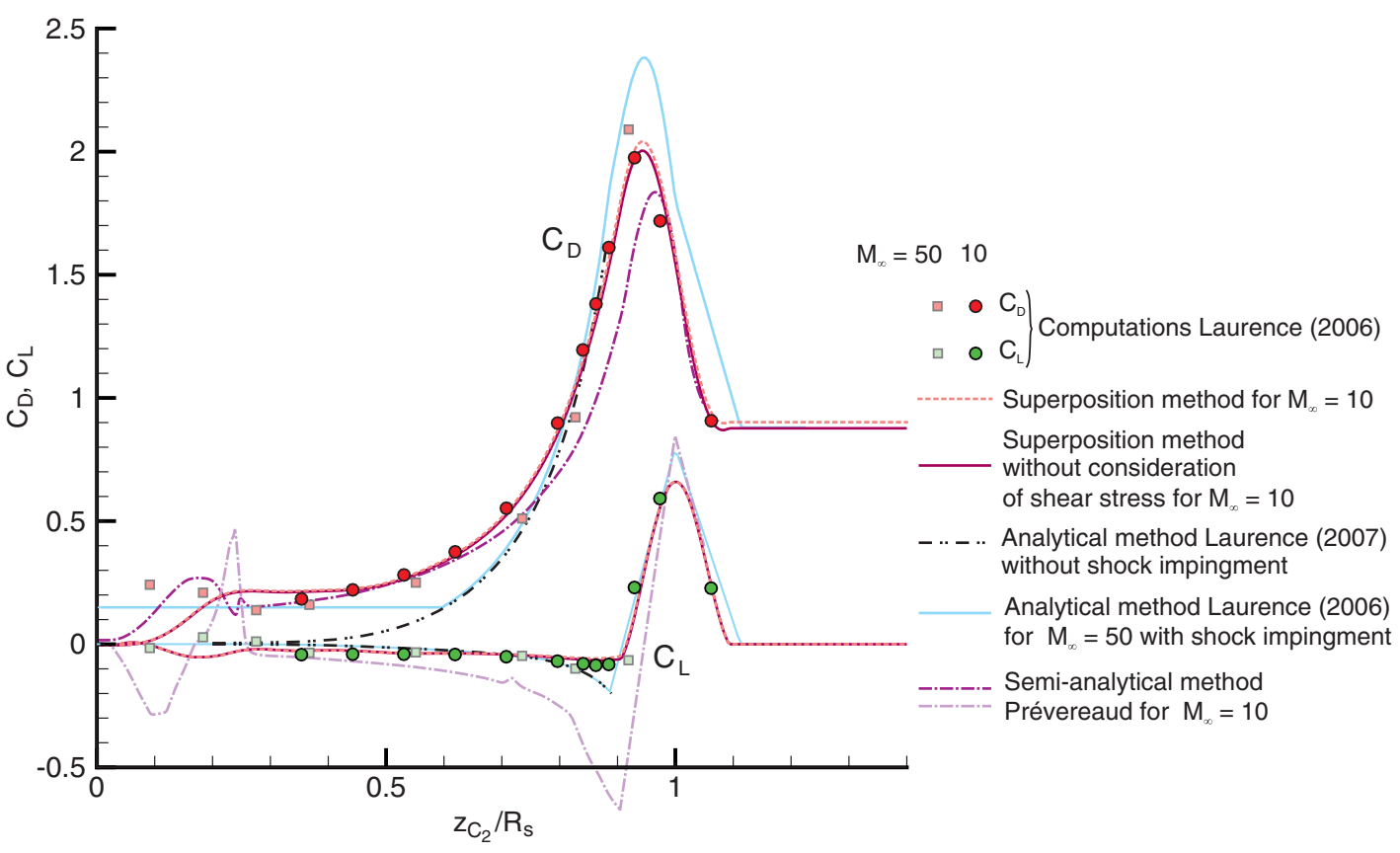

Fig. 10 Comparison of $C_{D}$ and $C_{L}$ values resulting from the superposition method, with computations with Cart3D for $M_{\infty}=10$, with AMROC computations for $M_{\infty}=10$ and $M_{\infty}=50$ by Laurence [6], with the Mach-number-independent analytical method of Ref. [7], the analytical method considering the impingment for $M_{\infty}=50$ from Ref. []ㅡ, and with the semi-analytical method for $M_{\infty}=10$ obtained from Ref. [9]: $x_{C_{2}} / d_{1}=1.5$, $d_{2} / d_{1}=1 / 4$.

sphere are shown in Figs. $\underline{9}$ and $\underline{10}$. They where calculated for $M_{\infty}=50$ in Ref. [6]. Comparing the results obtained with the analytical method with the computations for $M_{\infty}=50$ and comparing the results obtained with the superposition method with the computations for $M_{\infty}=10$ show that the curves of the superposition method match the $3 \mathrm{D}$ computations better than the curves of the analytical method for the cases in Figs. 9b and 10. Also, for the case of Fig. 9a, the superposition method matches the computed values better for most of the points, but at the maximum values of $C_{D}$ and $C_{L}$, the analytical method is more accurate.

The results of the superposition method are also compared to results obtained with the semi-analytical method by Prévereaud [9] in Figs. 9a, 9b, and 10. The results with the superposition method are more accurate than the results with the semi-analytical method.

Because of inaccuracies in the database for the subsonic regime, deviations of the results of the superposition method from 3D computations could be expected if the second sphere is located in the subsonic wake region for $x_{C_{2}} / d_{1} \lesssim 0.13$ in Figs. 9a and 9b. However, the curves calculated with the superposition method in those regions match the data points from the $3 \mathrm{D}$ computations quite well. The effect of the subsonic wake region is neglected completely by the analytical method as it does not model this region. In Ref. [6], $C_{D}$ was set to a constant value of 0.15 in the wake region for the calculations shown in Figs. 9 and 10, so no interaction phenomena are considered.

Viscous and unsteady effects could have important influence on the lift and drag of the second sphere in the subsonic wake of the first sphere and if strong shock-shock interactions occur for the bow shocks of the first and the second sphere. Therefore, to analyze the error of the superposition method in these regions, an analysis with unsteady, turbulent, 3D computations should be performed in the future.

The number of points of the grid of half of the second sphere was checked with a refinement study. $C_{D}$ and $C_{L}$ were calculated for 625 , $2500,10,000,40,000$, and 90,000 points. For all cases, the variation of the aerodynamic coefficients was large for less than 10,000 points. For $10,000,40,000$, and 90,000 points, the results for $C_{L}$ and $C_{D}$ were equal for all cases. Therefore, the solutions obtained with 90,000 points were independent of the number of points chosen. However, for the case with $x_{C_{2}} / d_{1}=4$ and $d_{2} / d_{1}=1 / 2$ in Fig. $9 \mathrm{~b}$ already 10,000 points would have been sufficient. The grid of the flow solution of the first sphere was strongly refined to ensure independence of the results of the superposition method from the grid of the flow solution of the first sphere. The axisymmetric grid contained 3.1 million cells.

\section{Detailed Comparison of Test Configuration}

For a deeper insight into the functioning of the superposition method, in this section, the results obtained with the superposition method for a test case of two spheres in hypersonic flow are compared to a $3 \mathrm{D}$ computation.

Figure 11a shows the configuration of the test case. The physical conditions are the same as in Table 1, except that the freestream Mach number is $M_{\infty}=7.0$. The diameters of the first and the second spheres are $d_{1}=d_{2}=0.05 \mathrm{~m}$, and the displacement of the second sphere in the $x$ direction is $x_{C 2}=0.1 \mathrm{~m}=2 d_{1}$ and in the $z$ direction is $z_{C 2}=0.08 \mathrm{~m}=1.6 d_{1}$. This configuration was chosen as a strong shock-shock interaction of the bow shocks of the first and the second spheres occurs for this configuration. The shock-shock interaction is not considered in the superposition method. Therefore, the configurations in which the bow shock of the first sphere impinges on the second sphere are the most critical cases. Furthermore, the largest deviations in the lift and drag coefficients in comparison to Cart3D computations and to the computations by Laurence [6] are found for large diameters and small axial displacement (see Sec. VII.A.1).

The 3D computation was carried out with the flow solver TAU. A symmetry plane was applied in the $x-z$ plane. The grid was refined with the TAU adaptation tool several times until grid independence was reached. The final grid contained 267.6 million cells. The final grid in the symmetry plane is shown in Fig. 11b. A steady, laminar flow was assumed. Unsteady phenomena that occurred due to the interaction of the flows around the two spheres were neglected. As for the supersonic and hypersonic simulations of the database, the second-order AUSMDV upwind scheme [22] was chosen for the spatial discretization.

A frequently used classification for shock-shock interactions for blunt bodies was developed by Edney [24]. In Fig. 12, the shockshock interaction of the bow shocks of the first and the second spheres is shown in more detail. The dashed line is the contour line for which $M=1$. The interaction is of Edney type IV. An oblique shock connects the bow shock of the second sphere outside and inside the region enclosed by the bow shock of the first sphere. A supersonic jet penetrates the subsonic region upstream of the second sphere. The 

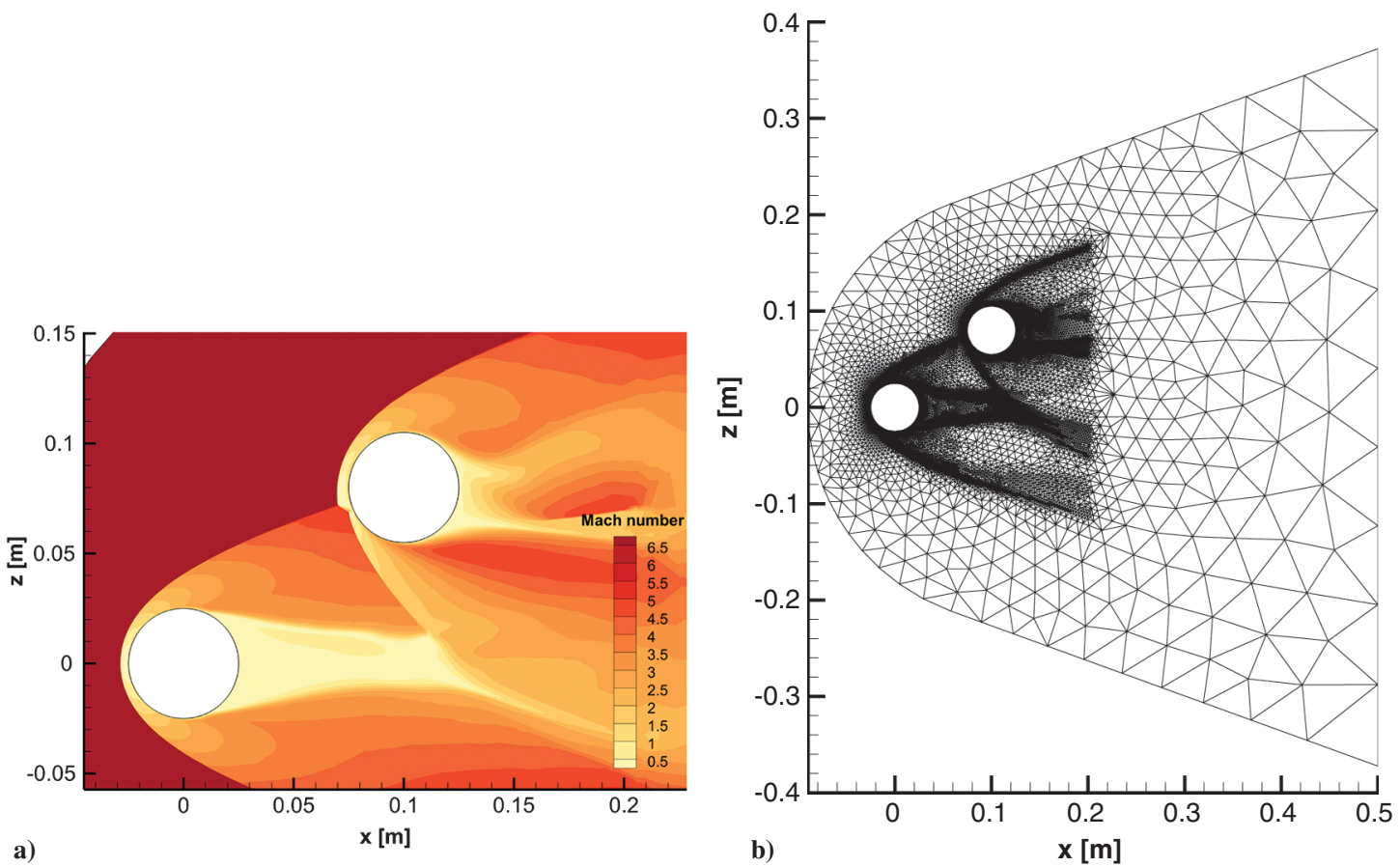

Fig. 11 3D computation of a test case for the validation of the superposition method: a) configuration and flowfield and b) final grid in the symmetry plane.

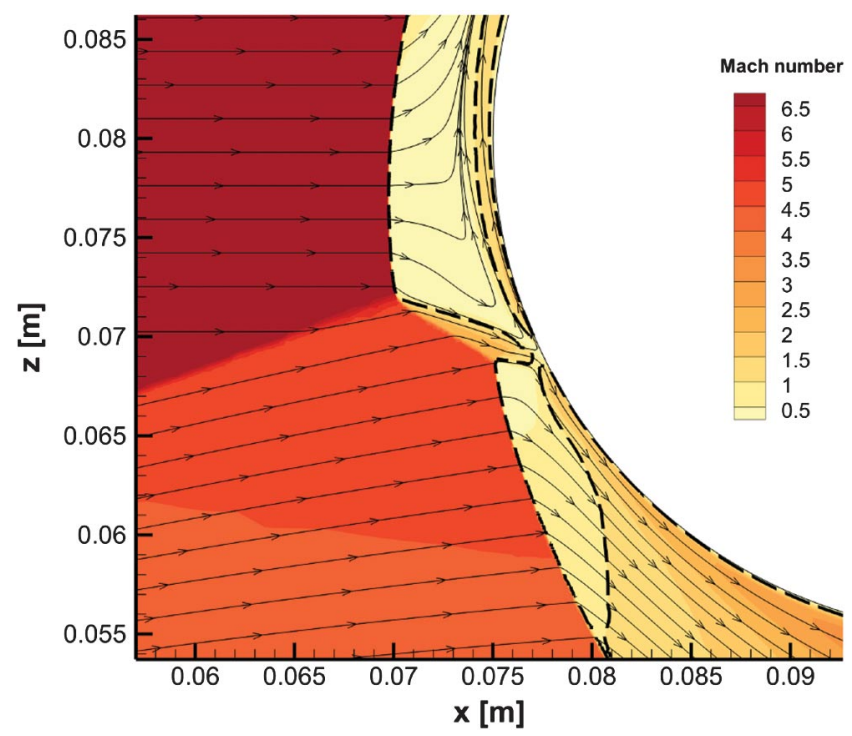

Fig. 12 Shock-shock interaction of the bow shocks of the first and the second spheres. The dashed line is $M=1$.

standoff distance of the bow shock of the second sphere in the inside region is smaller than in the freestream. As the Mach number in the inside region is smaller than in the freestream, neglecting the shock-shock interaction, the shock standoff distance is expected to be greater in the inside region than in the freestream. Therefore, the superposition method does not reproduce the flow phenomena correctly by neglecting the shock-shock interaction.

The aerodynamic coefficients for the second sphere, resulting from the $3 \mathrm{D}$ computation and from the superposition method, are shown in Table 2. The coefficients are divided in their components resulting from the pressure forces $C_{L_{p}}$ and $C_{D_{p}}$ and resulting from the shear stresses $C_{L_{r}}$ and $C_{D_{r}}$. Furthermore, the relative error and the absolute error of the coefficients, calculated with the superposition method with respect to the $3 \mathrm{D}$ computation, are given.

Section VII.A.1 showed that the superposition method overestimates the drag and the lift coefficient in the vicinity of the shock of the first sphere for large diameters of the second sphere and small downstream displacements. This is also found for the results of the TAU computation (see Table 2), but for the drag, the relative error of $3.6 \%$ is very small. The contribution of the pressure forces to the drag is much larger than the contribution of the forces resulting from the shear stress. $C_{D_{p}}$ is larger than $C_{D_{\tau}}$ by a factor of approximately 45 . Therefore, also the contribution of the error in $C_{D_{p}}$ to the error in the total drag coefficient $C_{D}$ is much larger than the contribution of the error in $C_{D_{\tau}}$. The drag resulting from the shear stress is slightly underestimated. Therefore, the relative error of the total drag coefficient is smaller than the relative error of the pressure drag coefficient.

The lift is overestimated by $23.3 \%$. Hence, the relative error in the lift is higher than the relative error in the drag. However, the absolute error is of the same order of magnitude. Also, for the lift, the contribution of the pressure forces to the lift is much larger than the contribution of the forces from the shear stress. Therefore, the absolute error of $C_{L_{\tau}}$ contibutes very little to the absolute error in $C_{L}$. The absolute error in $C_{L_{\tau}}$ is by a factor of approximately 10 smaller than the error in $C_{L_{p}}$. Nevertheless, the underestimation of $C_{L_{\tau}}$ counteracts the overestimation of $C_{L_{p}}$, so the error in $C_{L}$ is smaller than in $C_{L_{p}}$.

In the following, the pressure and the shear stress distribution on the second sphere are analyzed in detail. Figure 13a shows the pressure distribution on the second sphere from the $3 \mathrm{D}$ computation. In addition, contours of the logarithm of the pressure are shown for the flowfield in the $x-z$ plane in Fig. 13. The pressure distribution clearly shows a pressure peak arising from the supersonic jet penetrating the subsonic region. Furthermore, the skin friction lines

Table 2 Comparison of the lift and the drag coefficient resulting from the 3D computation and from the superposition method

\begin{tabular}{lcccc}
\hline \hline $\begin{array}{l}\text { Aerodynamic } \\
\text { coefficients }\end{array}$ & $\begin{array}{c}\text { 3D } \\
\text { computation }\end{array}$ & $\begin{array}{c}\text { Superposition } \\
\text { method }\end{array}$ & $\begin{array}{c}\text { Absolute } \\
\text { error }\end{array}$ & $\begin{array}{c}\text { Relative } \\
\text { error, \% }\end{array}$ \\
\hline$C_{D}$ & 1.164 & 1.206 & 0.04180 & 3.592 \\
$C_{D_{p}}$ & 1.139 & 1.182 & 0.04378 & 3.845 \\
$C_{D_{\tau}}$ & 0.02512 & 0.02314 & -0.001977 & -7.870 \\
$C_{L}$ & 0.1550 & 0.1910 & 0.03603 & 23.25 \\
$C_{L_{p}}$ & 0.1483 & 0.1901 & 0.04185 & 28.23 \\
$C_{L_{\tau}}$ & 0.006685 & 0.0008646 & -0.005821 & -87.07 \\
\hline \hline
\end{tabular}




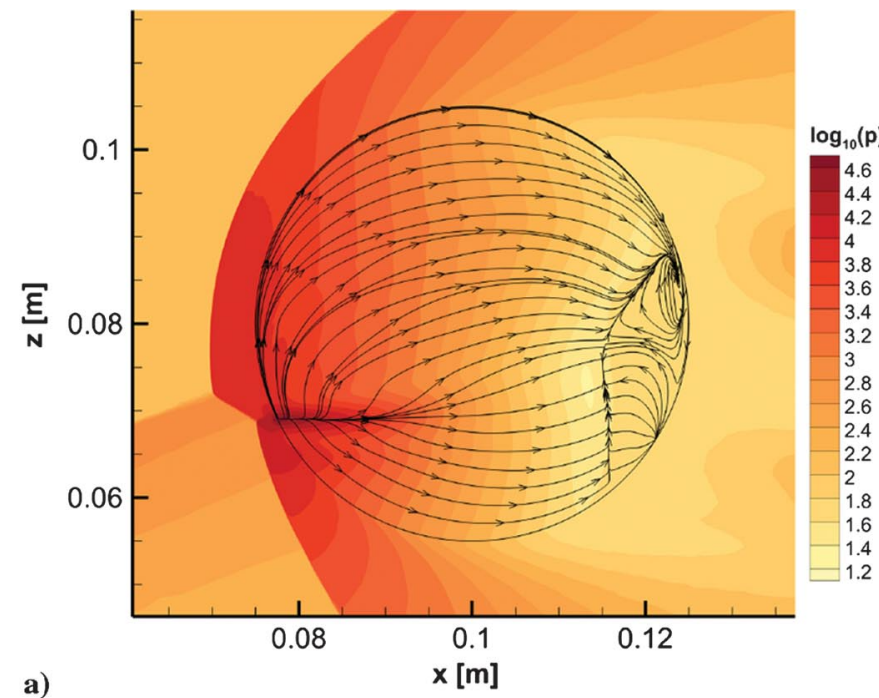

Fig. 13 Flow around the second sphere sphere from the superposition method.

are shown. The stagnation point is located where the supersonic jet impinges on the surface of the sphere.

Figure $13 \mathrm{~b}$ shows the pressure distribution on the sphere computed with the superposition method and the flow around the sphere taken from the 2D flow solution for the first sphere. The pressure distribution on the sphere is clearly divided by the bow shock of the first sphere. It changes abruptly from the freestream to the region enclosed by the bow shock of the first sphere. Also, the skin friction lines are shown for this case. The superposition method assumes the stagnation point to be the foremost point of the sphere in the freestream.

The comparison of Figs. 13a and $13 \mathrm{~b}$ shows that the pressure distribution of the superposition method on the part of the sphere outside of the bow shock of the first sphere shows good agreement with the pressure distribution of the 3D computation. Only in the wake region of the second sphere, the superposition method slightly overestimates the pressure. In the region enclosed by the bow shock of the first sphere, the difference between the pressure distributions is larger. The pressure peak in the computation is absent for the result of the superposition method. However, in other regions on the front side of the sphere, the pressure is overestimated. Here again, the pressure is slightly overestimated in the wake region of the second sphere.

Comparing the skin friction lines shows that the general shape of the lines is similar but that the location of the stagnation point is not reproduced correctly by the superposition method. Furthermore, the region where the skin friction lines are running upstream is larger for the superposition method than for the $3 \mathrm{D}$ computation. This indicates that the wake region where the flow separates from the sphere is larger for superposition method than for the $3 \mathrm{D}$ computation.

To analyze the pressure distribution in more detail, it is extracted for the two meridians on the sphere at $\theta=90 \mathrm{deg}$ and $\theta=270 \mathrm{deg}$. These are the upper and lower meridians in the $x-z$ plane. They are sketched in Fig. 14. The pressure distribution is shown in Fig. 15. In the area close to the foremost point of the sphere in the freestream ( $\phi=-90 \mathrm{deg}$ ), the superposition method overestimates the pressure on both meridians. This is due to the fact that the superposition method assumes this point to be the stagnation point, while its actual position on the $z$ axis is lower due to the shock-shock interaction.

As the meridian at $\theta=90 \mathrm{deg}$ lies mostly in the freestream, the influence of the shock-shock interaction on its pressure distribution is small. The superposition method overestimates the pressure on the front half of the sphere and in the area of the subsonic wake. On the back half of the sphere $(\phi>0 \mathrm{deg})$, the superposition method generates an increase in pressure in which the undisturbed shock of the first sphere intersects the surface of the second sphere (see Fig. 13b). The 3D computation shows that this region lies in the subsonic wake of the sphere (see Fig. 13a). Therefore, the increase in pressure cannot be observed in the computed solution. Figure $\underline{15}$

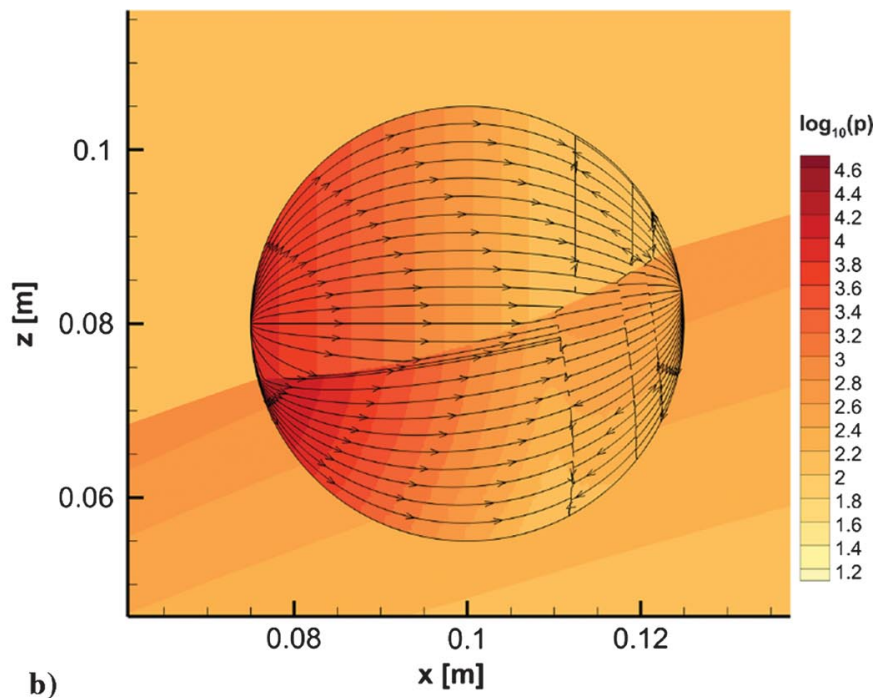
${ }_{10}(p)$ 

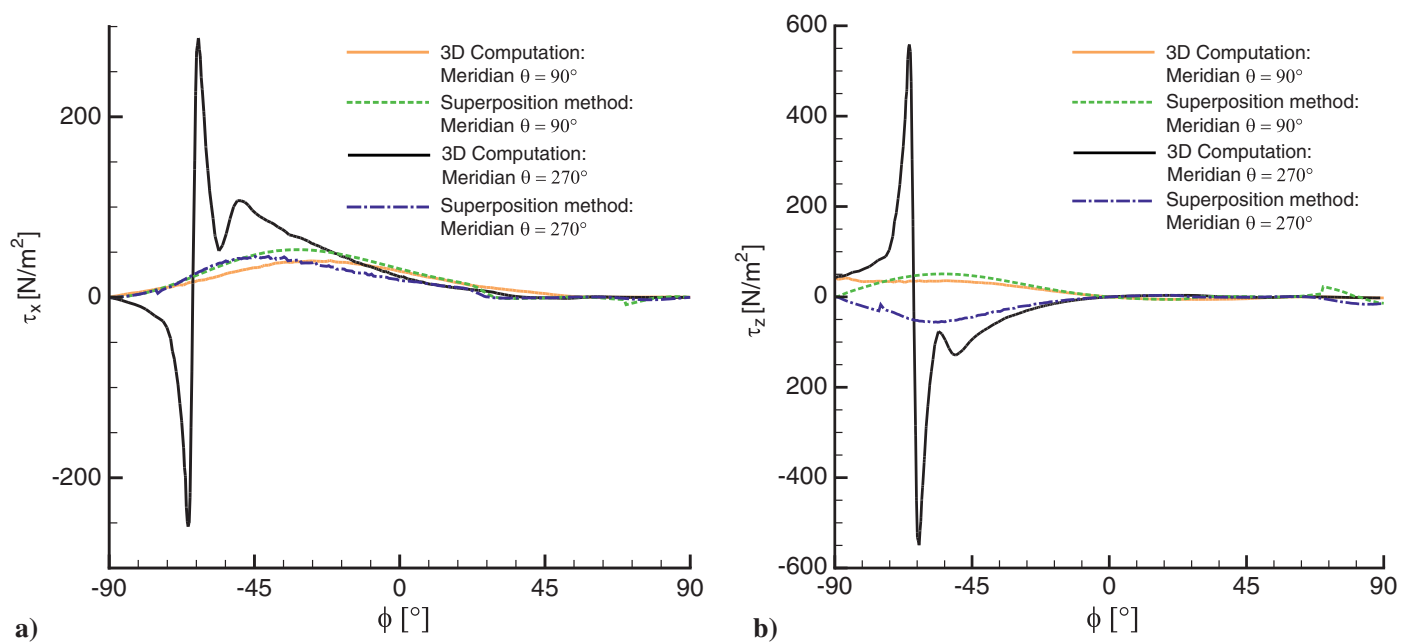

Fig. 16 Shear stress distributions of two meridians: a) $x$ direction and b) $z$ direction.

The computed pressure distribution of the meridian at $\theta=270 \mathrm{deg}$ shows two peaks, one at the impingement of the supersonic jet on the surface and a smaller one farther downstream. The pressure distribution of the superposition method shows a sharp increase at the impingement of the shock on the sphere. This increase is ahead of the pressure peak in the $3 \mathrm{D}$ computation as the point where the undisturbed shock impinges on the sphere is ahead of the point of impingement of the supersonic jet. On the back half of the sphere, the undisturbed shock does not intersect with this meridian again. Therefore, no jump in the pressure occurs here. Except for the pressure peaks in the computed solution, the superposition method generally overestimates the pressure.

Figure 15 shows that the deviation of the pressure in the front half is greater than in the back half due to the pressure peaks in the computed solution and due to the high overestimation of the pressure in this region for the bottom meridian $(\theta=270 \mathrm{deg})$. Again, the earlier separation for the superposition method and the resulting higher pressure in the wake region can be observed. However, the influence of the difference in the pressure distribution on the back half of the sphere on the total forces is negligible.

A small contribution to the forces on the sphere arises from the shear stresses. The shear stresses for the two meridians $\theta=90 \mathrm{deg}$ and $\theta=270 \mathrm{deg}$ in the $x$ and $z$ directions can be seen in Figs. 16a and 16b. For the upper meridian $(\theta=90 \mathrm{deg})$, the shape of the shear stresses resulting from the superposition method are similar to the computed solution for $\tau_{x}$ and $\tau_{z}$. Because of the location of the stagnation point below the $x_{k}$ axis for the bottom meridian $(\theta=270 \mathrm{deg})$, the shear stress of the computation jumps from negative to positive values for $\tau_{x}$ and from positive to negative values for $\tau_{z}$ along the meridian at the forward half of the sphere. This behavior is not caught by the superposition method. In general, the agreement of the superposition method with the computed solutions is poor for this meridian. As the superposition method does not describe the features of the shear stress distribution in a correct way for the shock-shock interaction, it cannot be stated that the superposition method predicts $C_{D_{\tau}}$ well in general. More test cases would be necessary to prove that the small error in $C_{D_{\tau}}$ is always guaranteed.

\section{Conclusion of Verification}

In this section, the superposition method was validated. Section VII.A.1 showed that for several test cases 3D computations can be reproduced very accurately. The analytical method developed in Refs. $[\underline{1}, \underline{6}-\underline{8}]$ is not valid in the wake region of the first sphere as the blast wave analogy does not cover this region. Additionally, the analytical method does not consider the recompression shock. Furthermore, the Newtonian pressure distribution used for the second sphere is only valid for high Mach numbers. The superposition method is probably more suitable to describe these interaction phenomena as fewer simplifications are made for the flow solution of the first sphere and the pressure distribution on the surface of the second sphere. Even though the semi-analytical method in Ref. [9] applies fewer simplifications, as the pressure in the flowfield of the first sphere is taken directly from numerical simulations and not from the blast wave analogy, the semi-analytical method is less accurate than the superposition method. In Sec. VII.A.2, a test case was compared in detail to a 3D computation, and it was shown where derivations of the results occur. Relatively large deviations occur locally for the pressure and the shear stress. However, the errors of the integrated values of $C_{D}$ and $C_{L}$ are small.

\section{B. Interaction Phenomena}

\section{Conditions for Interaction Phenomena}

From the aerodynamic coefficients follow the aerodynamic forces and consequently also the accelerations on the two spheres. Figure 17 shows the relative acceleration of the second sphere with respect to the first sphere $\Delta \boldsymbol{a}$, for the same relative position and diameter ratio of the first and the second sphere, as well as for the same Mach number as shown in Fig. 9b. The two spheres are of equal density and the mass of the first sphere $m_{1}$ is $1 \mathrm{~kg}$. The component of $\Delta \boldsymbol{a}$ in the direction of the freestream is $\Delta a_{x}$, and the component normal to the freestream is $\Delta a_{z}$. The horizontal line represents $\tan (\beta)$, where $\beta$ is the angle of the bow shock of the first sphere; $\beta$ is calculated with a modification of the correlation for the shock shape by Billig [25] proposed by Prévereaud [9] as she showed that her correlation fits the shock shape better than the correlation by Billig for high Mach numbers.

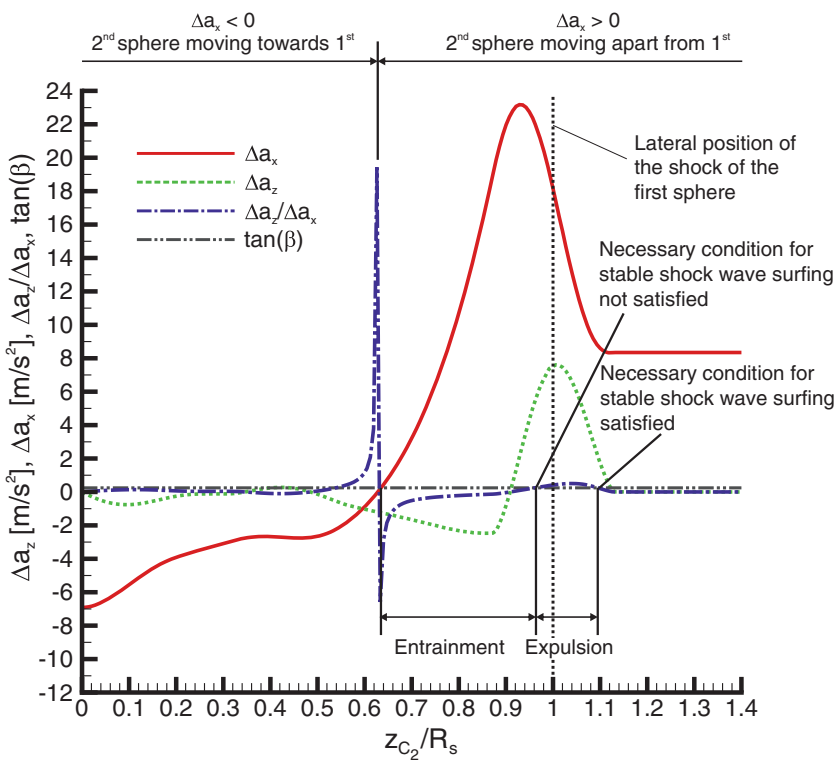

Fig. 17 Relative accelerations and interaction phenomena for spheres of the same density and $M=10, x_{C_{2}} / d_{1}=4, d_{2} / d_{1}=1 / 2$, and $m_{1}=1 \mathrm{~kg}$. 
The necessary condition for shock wave surfing described in Sec. II, that the acceleration on the sphere is tangential to the bow shock, can be written as

$$
\frac{\Delta a_{z}}{\Delta a_{x}}=\tan (\beta) \text { for } \Delta a_{x}>0
$$

This criterion also leads to more precise criteria for entrainment and expulsion of the second sphere. If, for $\Delta a_{x}>0, \Delta a_{z} / \Delta a_{x}$ is smaller than $\tan (\beta)$, entrainment will occur, and if it is larger than $\tan (\beta)$, the second sphere will be expelled. If $\Delta a_{x}<0$, the second sphere moves toward the first in the $x$ direction, and no shock wave surfing occurs.

Considering Eq. (3), shock wave surfing can occur at the intersection of the curve of $\Delta a_{z} / \Delta a_{x}$ with $\tan (\beta)$ for positive $\Delta a_{x}$. In Fig. 17, two points of intersection can be observed for which $\Delta a_{x}>0$ also holds. For stable shock wave surfing, an increase in $z_{C_{2}}$ has to result in a decrease in $\Delta a_{z} / \Delta a_{x}$; a decrease in $z_{C_{2}}$ has to result in an increase in $\Delta a_{z} / \Delta a_{x}$. This leads to the second necessary condition for shock wave surfing, which is that the slope of the function of $\Delta a_{z} / \Delta a_{x}=f\left(z_{C_{2}}\right)$ must be negative in the intersection point, and thus

$$
\frac{\partial\left(\Delta a_{z} / \Delta a_{x}\right)}{\partial z_{C_{2}}}<0
$$

Only one point of the two intersection points in Fig. 17 satisfies Eq. (4), which is at approximately $z_{C_{2}} / R_{s}=1.1$. The lateral distance of the second sphere $z_{C_{2}}$ with respect to the first sphere is scaled with the shock radius $R_{S}$ at $x_{C_{2}}$. Figure 17 shows that this point lies outside the region enclosed by the bow shock of the first sphere.

For the oblique shock generated by the leading sphere, the shock angle changes with the downstream displacement of the second sphere relative to the first one. Therefore, the intersection points of $\Delta a_{z} / \Delta a_{x}=\tan (\beta)$ found previously only show the locally stable behavior. Furthermore, Eqs. (3) and (4) are necessary but not sufficient conditions for shock wave surfing. For stable shock wave surfing to occur, the velocity vector of the second sphere has to be tangential to the bow shock of the first.

Also, the regions for entrainment $\left(\tan (\beta)<0, \Delta a_{x}>0\right)$, expulsion $\left(\tan (\beta)>0, \Delta a_{x}>0\right)$, and the second sphere moving toward the first $\left(\Delta a_{x}<0\right)$ can be observed in Fig. 17 .

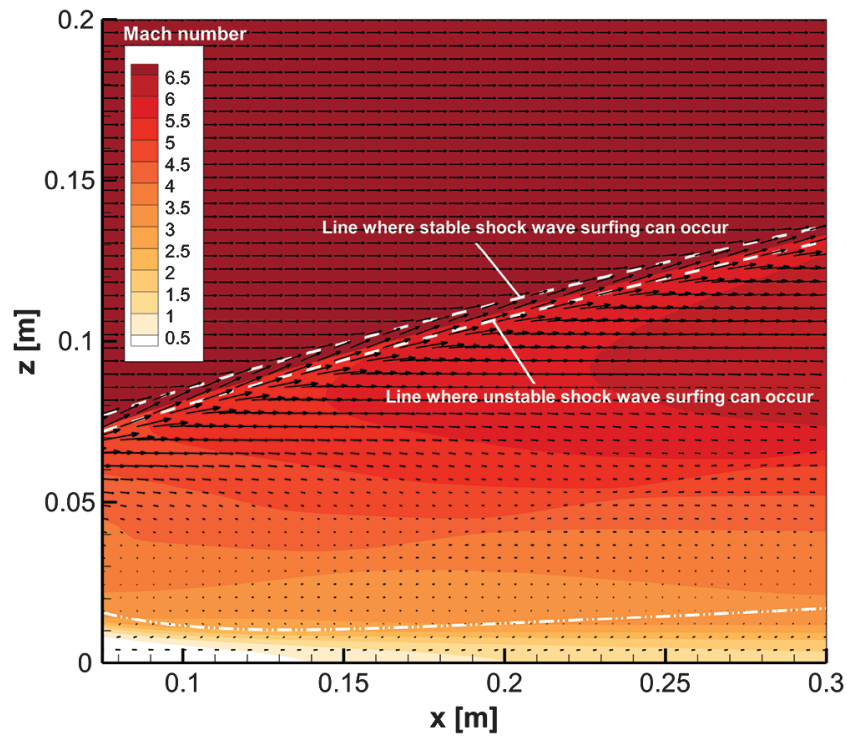

Fig. 18 Relative acceleration vectors $\Delta a$ of the second sphere and Mach number distribution of the flow of the first sphere for $d_{1}=0.025 \mathrm{~m}$, $d_{2}=1 / 6 d_{1}, \rho_{1}=\rho_{2}$, and $M_{\infty}=7.0$. Dashed lines are where shock wave surfing can occur; double dashed-double dotted lines are where $\Delta a_{x}=\mathbf{0}$.

\section{Analysis of Interaction Phenomena with Superposition Method}

In this subsection, the superposition method is applied to investigate the interaction of two spheres with $d_{2} / d_{1}=1 / 4$ at $M=7.0$. Section VII.A.1 showed that the $C_{D}$ and $C_{L}$ values calculated with the superposition method for $d_{2} / d_{1}=1 / 4$ are very close to 3D Euler computations even for low axial displacements of the second sphere with respect to the first sphere. Only pressure forces are taken into account. Both spheres are positioned in the $x-z$ plane.

Figure 18 shows vectors of the relative acceleration $\Delta \boldsymbol{a}$ of the second sphere with respect to the first sphere. The relative acceleration is normalized with the acceleration of the first sphere. Furthermore, Fig. 18 shows the Mach number distribution of the flowfield of the first sphere. In the freestream, the second sphere is accelerated downstream relative to the first sphere. In the wake region of the first sphere, the second sphere is accelerated upstream relative to the first sphere. The points where the necessary condition for shock wave surfing is met (see Eq. (3)) are shown with a dashed line. For the calculation of these lines, the shock wave angle $\beta$ was calculated with the correlation proposed by Prévereaud [9]. The vectors of relative accelerations are tangential to the bow shock on these lines.

Figures 19a and $19 \mathrm{~b}$ show the components of the $\Delta \boldsymbol{a}, \Delta a_{x}$, and $\Delta a_{z}$, normalized with the streamwise acceleration of the first sphere
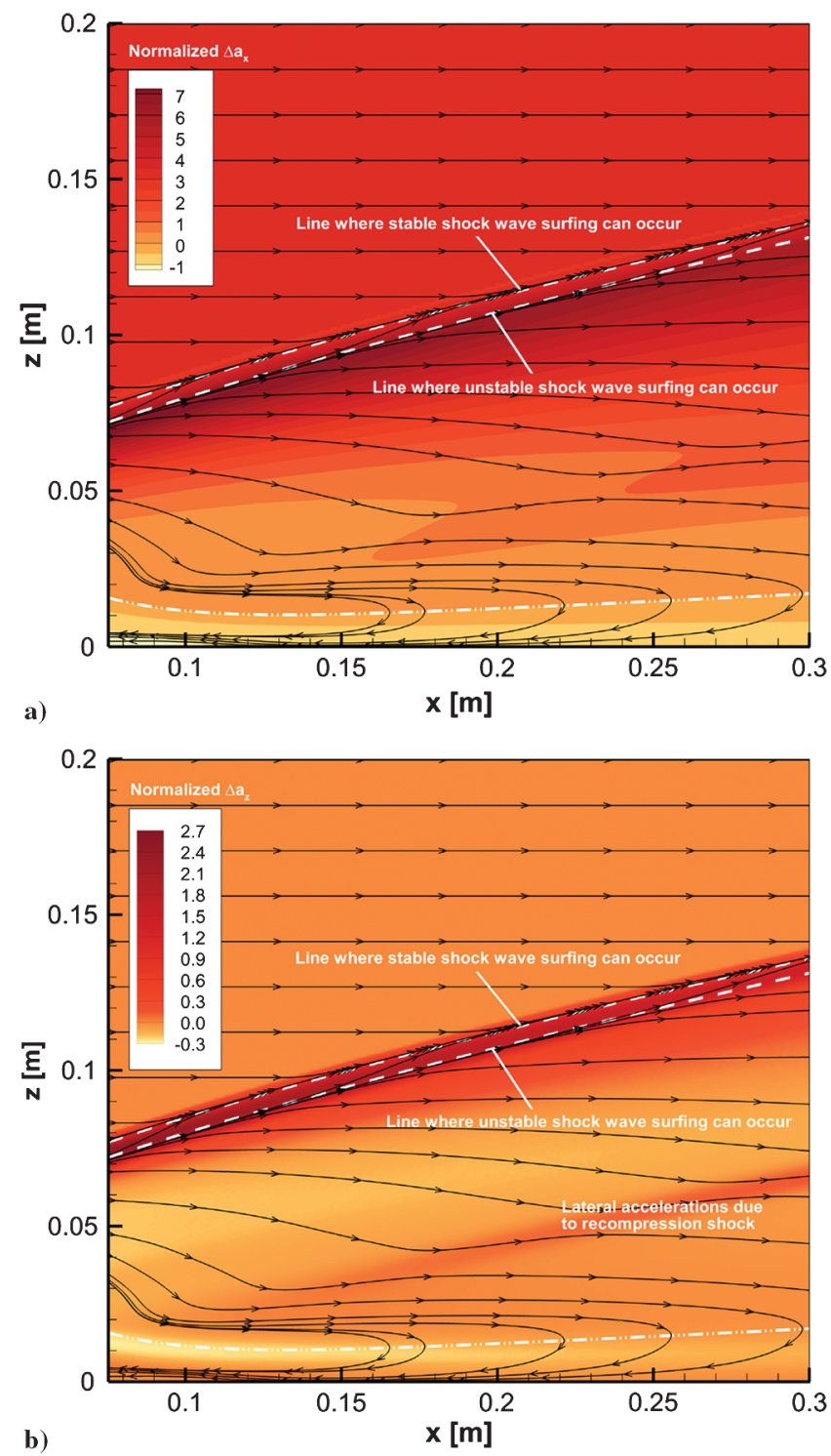

Fig. 19 Lines of relative acceleration of the second sphere for $d_{1}=0.025 \mathrm{~m}, d_{2}=1 / 4 d_{1}, \rho_{1}=\rho_{2}$, and $M_{\infty}=7.0$, normalized by the acceleration of the first sphere: a) $\Delta a_{x} / a_{x_{1}}$; b) $\Delta a_{z} / a_{x_{1}}$. Dashed lines are where shock wave surfing can occur; double dashed-double dotted lines are where $\Delta a_{x}=0$. 
$a_{x_{1}}$. Figure 19 has a resolution of $270 \times 200$ points (54,000 points) calculated with the superposition method. Furthermore, lines of the normalized relative acceleration are plotted. These lines are not trajectories of the second sphere but lines that are everywhere tangential to $\Delta \boldsymbol{a}$. To calculate the trajectories, the equations of motion need to be solved. Also, in Fig. 19, the points where shock wave surfing can occur are shown by the dashed lines.

From the necessary condition described by Eq. (4), it is expected that stable shock wave surfing can occur on the dashed line outside of the bow shock of the first sphere $\left(z_{C_{2}}>R_{S}\right)$ while the dashed line inside this region $\left(z_{C_{2}}<R_{S}\right)$ is expected to be unstable. This behavior can be proven by observing the acceleration lines. For the outside line, the acceleration lines converge with the dashed line, while for the inside dashed line, the acceleration lines diverge.

Figures $19 \mathrm{a}$ and $\underline{19 \mathrm{~b}}$ show that large axial and lateral accelerations occur in the region where Eq. (3) is satisfied. While the largest axial accelerations occur downstream of the line where unstable shock wave surfing can occur, the largest lateral accelerations occur between the two lines of possible shock wave surfing. Similar to the bow shock of the first sphere, also the recompression shock leads to an increase in the axial and lateral accelerations (see Figs. 19a and $19 \mathrm{~b}$ ). In the wake region, the acceleration lines run upstream. This corresponds to the drafting phenomenon described in Sec. II. The region in which the drafting phenomenon occurs is marked by the dash-dotted lines of $\Delta a_{x}=0$ in Figs. 18, 19a, and 19b.

The acceleration lines show that a second sphere located in the shocked region of the first sphere with no initial relative velocity with respect to the first sphere will be entrained. The 2250 configurations of the two spheres shown in Fig. 18 were computed with the superposition method in less than a day on a single core. Hence, using the superposition method is much faster than computing each configuration of the two spheres independently with a 3D computation. The results presented in this section were calculated with 10,000 points on the grid of half of the second sphere, as the drag and lift values calculated with the superposition method for $x_{C_{2}} / d_{1}=4$ and $d_{2} / d_{1}=1 / 2$ are already independent of the number of points for 10,000 points.

\section{Conclusions}

A method for the estimation of aerodynamic interactions of two bodies in supersonic and hypersonic flows has been proposed in this Paper. It is based on a superposition of flow solutions of a single body to compute the forces on the second body. Furthermore, conditions for the occurrence of the shock wave surfing phenomenon dependent on the relative accelerations of the second sphere have been described.

The method has been derived in detail for the case of two interacting spheres. A database of flow solutions of a single sphere for a range of Mach numbers has been created. The simulations in the database have been computed for axisymmetric, steady, and laminar flow. The database of the superposition method is exchangeable. Therefore, subsonic and transonic cases in the database that are currently calculated with the assumption of axisymmetric, steady, and laminar flow can be replaced by more sophisticated computations to improve the accuracy of the method at a later point in time. Expanding the database by a range of Reynolds numbers could lead to smaller errors in the superposition method, especially in the subsonic wake region of the first sphere.

Results obtained with the superposition method have been compared with 3D computations of configurations of two spheres. The error in the forces on the second sphere calculated with the superposition method are small. The method has been found to be a suitable tool to compute the forces on the spheres efficiently for a broad range of configurations. Also, effects that were not considered by existing analytical models, like the influence of the wake region of the first sphere and the corresponding recompression shock on the forces on the second sphere, are taken into account. Interaction phenomena in dependence of the relative acceleration of the spheres can be determined and reproduced with this method.

The method of the superposition of flowfields is not limited to spheres. An expansion to nonspherical shapes would require some modifications in the code and additional degrees of freedom in the database.

The forces computed with the superposition method allow the computation of trajectories of interacting bodies by solving the equations of motion. The method has been developed for the design of wind tunnel experiments. Additionally, it could be applied to estimate the forces on the fragments of meteoroids and space debris during supersonic or hypersonic atmospheric entry. For this application, the method would have to be expanded to describe the interaction of more than two bodies.

The strewn field of meteoroids and space debris on the Earth's surface can be determined more accurately by considering the interaction phenomena in the trajectory calculations of their fragments. Furthermore, the assessment of ground damage caused by a meteoroid can be improved by considering the interaction phenomena in the calculations of its energy deposition rates in the atmosphere and its airburst altitude.

\section{References}

[1] Laurence, S. J., and Deiterding, R., "Shock-Wave Surfing," Journal of Fluid Mechanics, Vol. 676, June 2011, pp. 396-431. doi: $10.1017 / \mathrm{jfm} .2011 .57$

[2] Artem'eva, N. A., and Shuvalov, V. V., "Motion of a Fragmented Meteoroid Through the Planetary Atmosphere," Journal of Geophysical Research: Planets, Vol. 106, No. E2, 2001, pp. 3297-3309. doi:10.1029/2000JE001264

[3] Passey, Q. R., and Melosh, H. J., "Effects of Atmospheric Breakup on Crater Field Formation," Icarus, Vol. 42, No. 2, 1980, pp. 211-233. doi:10.1016/0019-1035(80)90072-X

[4] Wheeler, L. F., Register, P. J., and Mathias, D. L., "A Fragment-Cloud Model for Asteroid Breakup and Atmospheric Energy Deposition," Icarus, Vol. 295, Oct. 2017, pp. 149-169. doi:10.1016/j.icarus.2017.02.011

[5] Register, P. J., Mathias, D. L., and Wheeler, L. F., "Asteroid Fragmentation Approaches for Modeling Atmospheric Energy Deposition," Icarus, Vol. 284, March 2017, pp. 157-166. doi:10.1016/j.icarus.2016.11.020

[6] Laurence, S. J., "Proximal Bodies in Hypersonic Flow," Ph.D. Thesis, California Inst. of Technology, Pasadena, CA, 2006.

[7] Laurence, S. J., Deiterding, R., and Hornung, G., "Proximal Bodies in Hypersonic Flow," Journal of Fluid Mechanics, Vol. 590, Nov. 2007, pp. 209-237. doi:10.1017/S0022112007007987

[8] Laurence, S. J., Parziale, N. J., and Deiterding, R., "Dynamical Separation of Spherical Bodies in Supersonic Flow," Journal of Fluid Mechanics, Vol. 713, Dec. 2012, pp. 159-182. doi: $10.1017 / \mathrm{jfm} .2012 .453$

[9] Prévereaud, Y., "Contribution à la Modélisation de la Rentrée Atmosphérique des Débris Spatiaux," Ph.D. Thesis, Inst. Supérieur de l'Aéronautique et de l'Espace, Toulouse, France, 2014.

[10] Aftosmis, M. J., Berger, M. J., and Melton, J. E., "Robust and Efficient Cartesian Mesh Generation for Component-Based Geometry," AIAA Journal, Vol. 36, No. 6, 1998, pp. 952-960. doi: $10.2514 / 2.464$

[11] Aftosmis, M. J., Berger, M. J., and Adomavicius, G., "A Parallel Multilevel Method for Adaptively Refined Cartesian Grids with Embedded Boundaries," 38th Aerospace Sciences Meeting and Exhibit, AIAA Paper 2000-0808, 2000. doi: $10.2514 / 6.2000-808$

[12] Nemec, M., and Aftosmis, M. J., "Toward Automatic Verification of Goal-Oriented Flow Simulations," NASA Ames Research Center TR NAS-2014-04, 2014.

[13] Schultz, P. H., and Sugita, S., "Penetrating and Escaping the Atmospheres of Venus and Earth," Lunar and Planetary Science Conference, Vol. 25, Lunar and Planetary Inst., Houston, 1994, pp. 1215-1216.

[14] Artem'eva, N. A., and Shuvalov, V. V., "Interaction of Shock Waves During the Passage of a Disrupted Meteoroid Through the Atmosphere," Shock Waves, Vol. 5, No. 6, 1996, pp. 359-367. doi:10.1007/BF02434011

[15] Boroviĉka, J., and Kalenda, P., "The Morávka Meteorite Fall: 4. Meteoroid Dynamics and Fragmentation in the Atmosphere," Meteoritics \& Planetary Science, Vol. 38, No. 7, 2003, pp. 1023-1043. doi:10.1111/maps.2003.38.issue-7

[16] Anderson, J. D., Jr., Hypersonic and High Temperature Gas Dynamics, 2nd ed., AIAA, Reston, VA, 2006, pp. 55-63. doi: $10.2514 / 4.861956$ 
[17] Schwamborn, D., Gerhold, T., and Heinrich, R., "The DLR TAU-Code: Recent Applications in Research and Industry," ECCOMAS CFD 2006 Conference, TU Delft, Delft, The Netherlands, 2006.

[18] Langer, S., Schwöppe, A., and Kroll, N., "The DLR Flow Solver TAU—Status and Recent Algorithmic Developments," 52nd Aerospace Sciences Meeting, AIAA Paper 2014-0080, 2014. doi:10.2514/6.2014-0080

[19] Gülhan, A., Willems, S., and Schleutker, T., "Multibody Aerothermodynamics of Space Debris Fragments," VKI Lecture Series STO-AVT-262. Space Debris Reentry and Mitigation, von Kármán Inst. for Fluid Dynamics, Sint-Genesius-Rode, Belgium, 2016, pp. 10.1-10.26.

[20] Naumann, A., "Luftwiderstand von Kugeln bei Hohen Unterschallgeschwindigkeiten," Allgemeine Wärmetechnik, Vol. 4, 1953, pp. 217-221.

[21] Schlichting, H., and Gersten, K., Grenzschicht-Theorie, 10th ed., Springer-Verlag, Berlin, 2006, pp. 8-10, 22. doi:10.1007/3-540-32985-4

[22] Liou, M., and Wada, Y., "A Flux Splitting Scheme with High-Resolution and Robustness for Discontinuities," 32nd Aerospace Sciences Meeting and Exhibit, AIAA Paper 1994-0083, 1994.

doi: $10.2514 / 6.1994-83$

[23] Deiterding, R., "Block-Structured Adaptive Mesh RefinementTheory, Implementation and Application," ESAIM: Proceedings, Vol. 34, 2011, pp. 97-150 doi:10.1051/proc/201134002

[24] Edney, B., "Anomalous Heat Transfer and Pressure Distributions on Blunt Bodies at Hypersonic Speeds in the Presence of an Impinging Shock," Flygtekniska Forsoksanstalten, TR-115, Stockholm, Sweden, 1968

[25] Billig, F. S., "Shock-Wave Shapes Around Spherical- and CylindricalNosed Bodies," Journal of Spacecraft and Rockets, Vol. 4, No. 6, 1967, pp. 822-823.

doi: $\underline{10.2514 / 3.28969}$

M. S. Miller Associate Editor 